Please quote as: Dünnebeil, S.; Sunyaev, A.; Leimeister, J. M. \& Krcmar, H. (2013): Modulare Softwarearchitektur für Mehrwertanwendungen der deutschen Gesundheitstelematik. In: Wirtschaftsinformatik (WI), Ausgabe/Number: 1, Vol. 55, Erscheinungsjahr/Year: 2012. Seiten/Pages: 3-18. 


\section{Modulare Softwarearchitektur für Mehrwertanwendungen der deutschen Gesundheitstelematik}

Elektronische Gesundheitskarte bzw. Telematikinfrastruktur sind ein vieldiskutiertes Thema in der deutschen Öffentlichkeit. Die Anwendungen, die die Dienste der serviceorientierten Gesundheitstelematik nutzen sollen, um Verbesserungen in das Gesundheitswesen zu tragen, wurden jedoch bisher kaum thematisiert. Der Artikel diskutiert Potenziale solcher Mehrwertanwendungen, die nicht den Beschränkungen bisheriger Informationssysteme in medizinischen Institutionen unterliegen und neue Potenziale eröffnen. Zu diesem Zweck werden übergreifende Anforderungen an Mehrwertanwendungen in eine wiederverwendbare Softwarearchitektur überführt. Die Implementierung einer Mehrwertanwendung wird auf Basis der Architektur illustriert, indem eine kooperative Anwendung zur Überweisungs- und Terminverwaltung implementiert wird. Die prominenten Themen Sicherheit, Interoperabilität sowie Patienten- und Systemintegration nehmen dabei eine herausragende Rolle ein.

\section{Die Autoren}

Sebastian Dünnebeil, M.Sc.

Prof. Dr. Helmut Krcmar

Lehrstuhl für Wirtschaftsinformatik

Fakultät für Informatik

Technische Universität München

Boltzmannstr. 3

85748 Garching bei München

Deutschland

sebastian.duennebeil@in.tum.de

krcmar@in.tum.de

Jun.-Prof. Dr. Ali Sunyaev ( $\varangle$ )

Juniorprofessur für

Wirtschaftsinformatik und

Information Systems Quality

Wirtschafts- und

Sozialwissenschaftliche Fakultät

Universität zu Köln

Pohligstr. 1

50969 Köln

Deutschland

sunyaev@wiso.uni-koeln.de url: http://www.isq.uni-koeln.de
Prof. Dr. Jan Marco Leimeister Fachgebiet Wirtschaftsinformatik Fachbereich

Wirtschaftswissenschaften

Universität Kassel

Nora-Platiel-Str. 4 Raum 1102

34127 Kassel

Deutschland

und

Institut für Wirtschaftsinformatik (IWI

HSG)

Universität St. Gallen

St. Gallen

Schweiz

JanMarco.Leimeister@unisg.ch

Eingegangen: 2012-03-01

Angenommen: 2012-10-06

Angenommen nach zwei Überarbeitungen durch Prof. Dr. Kirn.

This article is also available in English via http://www.springerlink.com and http://www.bise-journal.org: Dünnebeil S, Sunyaev A, Leimeister JM, Krcmar H (2012) Modular Architecture of Value-Added Applications for German Healthcare Telematics. Electronic Health card, Healthcare telematics, Value-added applications, Value-added services, Software architecture, Design research. Bus Inf Syst Eng. doi: 10.1007/s12599-012-0243-3.
Zusätzliche Information ist in der Online-Version dieses Beitrags (doi: 10.1007/s11576-012-0345-z) enthalten.

(C) Springer Fachmedien Wiesbaden 2013

\section{Einleitung}

Die Diskussion um die Einführung der elektronischen Gesundheitskarte (eGK) und der damit einhergehenden einheitlichen Vernetzung des deutschen Gesundheitswesens schwelt in der deutschen Öffentlichkeit seit mehreren Jahren. Das ursprünglich geplante Einführungsdatum, das Jahr 2006, ist seit langem verstrichen, die Vernetzung der Leistungserbringer im Gesundheitswesen lässt weiter auf sich warten (Tuffs 2010). Speziell seitens der Ärzte gibt es vehementen Widerstand gegen die laufenden Aktivitäten zum Aufbau der deutschen Telematikinfrastruktur (TI). Durch die Nutzung der von staatlicher Seite spezifizierten Dienste der eGK befürchten Leistungserbringer zusätzlichen Arbeitsaufwand und finanzielle Mindereinnahmen (Allensbach 2010), ohne dass dafür im Gegenzug ein adäquater medizinischer Zusatznutzen in den medizinischen Kernprozessen 
bereitgestellt wird (Kirn 2005; Mertens 2012). Auf große Ablehnung stieß primär die dauerhafte, zentrale Speicherung von Patientendaten in elektronischen $\mathrm{Pa}$ tientenakten (Dünnebeil et al. 2010b). Der Deutsche Ärztetag, das zentrale Gremium der hiesigen Ärzteschaft, lehnte das Projekt in allen Abstimmungen ab und forderte eine grundlegende Neuausrichtung der Initiative, um die Anforderungen der Ärzte hinreichend zu berücksichtigen (BITKOM 2009). Im Jahre 2009 wurden die Verantwortlichkeiten für die eGK an medizinische Fachgesellschaften übergeben, um diese im Sinne der Leistungserbringer neu auszurichten.

Gleichzeitig zeichnet sich in der deutschen Gesundheitsversorgung ein „[... Übergang vom derzeit dominierenden plan- und kollektivwirtschaftlichen Steuerungsregime hin $\mathrm{zu}$ einer konsequent wettbewerblichen Ausgestaltung der individuellen Vertragsbeziehungen zwischen Krankenkassen und Leistungsanbietern [...]" ab (Jacobs und Schulze 2004). Individuell ausgestaltete Prozesse sollen die Gesundheitsversorgung zwischen Patienten, Leistungserbringern und Kostenträgern fallbezogen organisieren und benötigen hierfür vermehrt Unterstützung durch vernetzte Informationssysteme (IS). Diese wurden in den Bemühungen zur Spezifikation der Telematik bisher nicht berücksichtigt. Fallspezifische Anforderungen und Versorgungsprozesse, wie sie in integrierten Versorgungskonzepten angedacht wurden (Silber 2006), können mit den gegenwärtig spezifizierten Telematikdiensten nicht hinreichend abgebildet werden. Die staatlichen Anwendungen der TI orientieren sich bisher vielmehr an den kollektiven Versorgungsprozessen der gesetzlichen Regelversorgung.

Mehrwertanwendungen (MWA) der eGK werden nicht von staatlichen Institutionen spezifiziert. Sie sollen vielmehr informationstechnische Innovationen in den Institutionen der Leistungserbringer ermöglichen, die von unabhängigen Firmen, Krankenkassen oder Forschungsinstituten auf Basis der TI entwickelt werden (Neuhaus et al. 2006; Rohner und Winter 2008). Als Vorbild können Anwendungen für mobile Endgeräte angesehen werden, die von einer verteilten, weltweiten Entwicklergemeinde erstellt werden und für viele Nutzer einen Mehrwert darstellen (Holzer und Ondrus 2011). Offen ist, wie ähnliche Potenziale für MWA rund um die eGK zu heben sind, indem Ansätze für die TI er- arbeitet werden, die eine vertrauenswürdige Implementierung von praxisnahen Nutzenszenarien zulässt. Betrachtet man den Widerstand gegen die von staatlicher Seite spezifizierten Dienste der eGK, so ist es langfristig eine essenzielle Aufgabe für die Konzeption von MWA der TI, eine Referenzarchitektur bereitzustellen, die eine hohe Akzeptanz bei den Nutzergruppen genießt. Mit der Aufnahme der Hauptkritikpunkte von Ärzten in eine Softwarearchitektur für Anwendungen der TI, bei gleichzeitiger Bereitstellung von praxisnahen Nutzenszenarien, sollte den berechtigen Sorgen der Ärzte Rechnung getragen werden, um für eine hohe Akzeptanz sorgen. Eine modularisierte Referenzarchitektur kann ferner die Spezifikation, Implementierung und Wiederverwendung wohldefinierter Komponenten vorantreiben.

Ziel dieses Beitrags ist es, die Relevanz von MWA für das deutsche Gesundheitswesen zu zeigen, eine modulare Softwarearchitektur für MWA der TI, die den Anforderungen von Leistungserbringern gerecht wird, $\mathrm{zu}$ erarbeiten und deren Handhabbarkeit und Eignung anhand einer Beispielimplementierung zu skizzieren. Dabei sollen, neben einer Architekturspezifikation, Aussagen über sinnvolle Herangehensweisen an die Entwicklung von MWA der eGK für das deutsche Gesundheitswesen erarbeitet werden, die langfristig in ein umfassendes Referenzmodell münden können. Speziell die Kommunikation zwischen verteilten Anwendungen (bspw. in Arztpraxen), die keine übergreifende Projektsteuerung haben, soll dargestellt werden. Im Gesundheitswesen werden - speziell bei fehlenden und kleinen IT-Abteilungen - Architekturentscheidungen tendenziell vernachlässigt (Köbler et al. 2010). Wir adressieren daher die folgenden Forschungsfragen:

- Welche übergreifenden Anforderungen ergeben sich für MWA der eGK?

- Welche modulare Architektur eignet sich zur strukturierten Umsetzung der übergreifenden Anforderungen?

- Welche Implikationen ergeben sich aus der Implementierung einer Beispielanwendung?

\subsection{Status quo von \\ Arztinformationssystemen im deutschen Gesundheitswesen}

Seit Jahrzehnten nutzten niedergelassene Haus- und Fachärzte Arztinformationssysteme (AIS), die sie bei der medizinischen und administrativen Abwicklung ihrer Behandlungen unterstützen. Seit dem 1. Januar 2011 ist eine Onlineabrechnung für alle niedergelassenen Ärzte durch ein zugelassenes System der Kassenärztlichen Bundesvereinigung (KBV) vorgeschrieben (KBV 2010). Zum Verfassungszeitpunkt des Artikels (Q4/2011) waren 156 verschiedene Systeme von der KBV zur Abrechnung zugelassen (KBV 2011a). AIS verfügen heute über eine Reihe von zusätzlichen Funktionen wie Terminverwaltung, Patientenakte oder Arzneimitteldatenbank und sind im täglichen Ablauf der niedergelassenen Ärzte ein elementarer Bestandteil der täglichen Arbeit geworden (Dünnebeil et al. 2010a). Eine strukturierte medizinische Kommunikation zwischen den Systemen verschiedener Anbieter ist bis dato jedoch kaum etabliert. Da im Gesundheitswesen auch institutionsübergreifende Prozesse existieren, die der Unterstützung durch IT-Systeme bedürfen, wurde das Projekt der eGK in Deutschland gestartet (BMG 2005). Um einen sicheren, interoperablen Austausch von Patientendaten zwischen den Systemen der Leistungserbringer zu ermöglichen, wurde 2004 begonnen, eine übergreifende TI zu spezifizieren (Fraunhofer 2005).

\subsection{Die deutsche Telematikinfrastruktur}

Die deutsche TI folgt dem Paradigma der serviceorientierten Architekturen (SOA). Es werden Austauschdienste für medizinische Daten als XML-Webservices angeboten (Abb. 1). Die Systeme der Akteure im Gesundheitswesen sind als Nutzer dieser Dienste vorgesehen (gematik 2009a). Leistungserbringer können die Webservices der eGK im Rahmen einheitlicher und universell verfügbarer Spezifikationen der gematik, der Gesellschaft für Telematikanwendungen der Gesundheitskarte (gematik), aufrufen (gematik 2012). So sollen einheitliche Kommunikationsstandards etabliert werden, die Interoperabilität, Sicherheit und die Einhaltung der gesetzlichen Rahmenbedingungen garantieren. Eine aktuelle detaillierte technische Beschreibung der deutschen Telematikinfrastruktur findet man bei Dehling und Sunyaev (2012).

Die Dienste der TI können in drei Klassen aufgeteilt werden:

Die TI stellt Basisdienste als lokale oder zentrale Webservices zur Verfügung, die es ermöglichen, einheitliche Verfahren zur Authentifizierung, Autorisierung, Signatur und Verschlüsselung in den Anwendungen zu nutzen. Die Basisdienste 


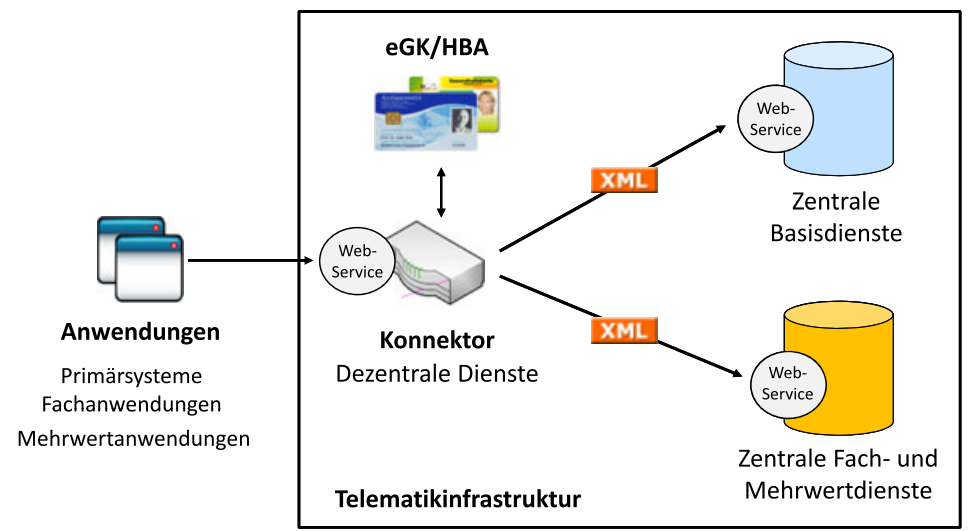

Abb. 1 Telematikinfrastruktur

werden von der gematik spezifiziert, garantieren ein übergreifendes Sicherheitsniveau und erfüllen gesetzliche Richtlinien, wie Signaturbefähigung und Sichtbarkeit von Patientendaten. Alle Dienste sind über einen Konnektor erreichbar, der die Schnittstellen als vordefinierte Webservices anbietet. In Arztpraxen ist dieser Konnektor eine Hardwarekomponente mit einem eingebetteten System. Der Konnektor stellt lokale Dienste, wie Verschlüsselung, Digitale Signatur oder Kartenmanagement, bereit und verbindet sich bei Bedarf mit den zentralen Diensten der TI (gematik 2009b).

Auch medizinische und administrative Fachdienste werden von der gematik spezifiziert. Ihre Nutzung kann verpflichtend sein, wie der Abgleich von Versichertenstammdaten, oder freiwillig, wie das Anlegen eines Notfalldatensatzes. Als freiwilliger Fachdienst wurde in den Architekturspezifikationen auch eine elektronische Patientenakte vorgesehen, die allen autorisierten Ärzten medizinische Daten eines Patienten über einen zentralen Dienst zur Verfügung stellt (gematik 2009a). Für einen solchen Fachdienst liegen bisher keine Spezifikationen vor. Die Realisierung dieses Projektes ist wegen geringer Akzeptanz ungewiss (Allensbach 2010).

Mehrwertdienste (MWD) der TI können von beliebigen Organisationen spezifiziert werden und sollen für eine oder mehrere Nutzergruppen der Gesundheitstelematik, z. B. Patienten, Ärzte, Pfleger oder Apotheker, einen Mehrwert bereitstellen. Die KBV wurde mit der Spezifikation eines MWD zur Kommunikation zwischen Leistungserbringern beauftragt. Er soll eine sichere Kommunikation von einheitlichen Arztbriefen zwischen den Institutionen des Gesundheitswesens ermöglichen und nutzt dabei u.a. die Telematikdienste zur Digitalen Signatur, Verschlüsselung oder den Verzeichnisdienst. Weitere MWD sind möglich, um andere Funktionen bereitzustellen, die zur strukturierten Behandlung benötigt werden (VHitG 2006). MWD sollen laut der Wirtschaftlichkeitsanalyse langfristig, mit bis zu 2,5 Mrd. Euro jährlichen Einsparungen, den größten finanziellen Nutzen zur TI beisteuern (Bernnat 2006).

\subsection{Dienstnutzer der TI}

Zum Zeitpunkt der Spezifikation (2004) der eGK war die Verbreitung von Anwendungen auf ubiquitären und mobilen Endgeräten noch nicht sehr verbreitet, sie wurden daher nicht als typische Nutzer der TI angesehen. Als Konsumenten für die beschriebenen Dienste der eGK wurden ursprünglich nur die Primärsysteme der Leistungserbringer in Krankenhäusern, Apotheken und Arztpraxen vorgesehen (gematik 2009a). Als Primärsysteme werden medizinische IS bezeichnet, die wichtige Behandlungsabläufe in Institutionen des Gesundheitswesens unterstützen. In der Regel werden sie von einem spezialisierten Hersteller als proprietäres Produkt bereitgestellt. Speziell in kleinen Institutionen, z.B. Arztpraxen und Apotheken, deckt dieses jedoch nur einen standardisierten Funktionsumfang ab (TURBOMED 2012). Es ist nicht absehbar, ob eine modulare Erweiterung dieser Systeme in $\mathrm{Zu}-$ kunft angestrebt wird, um sie einer breiteren Basis von Entwicklern zu öffnen. Die standardisierte Behandlung von Patienten, die einem einheitlichen Ablauf folgt und durch Standardsoftware hinreichend abgebildet werden kann, gehört jedoch in vielen Fällen der Vergangenheit an. Integrierte Versorgungsverträge, die nach $\$ 140$ a des Fünften Buchs des Sozialgesetzbuchs (SGB5) zwischen Krankenkassen und Ärzteverbünden geschlossen werden können (BRD 2004b), richten sich nach spezifischen Anforderungen einer Region oder Patientenpopulation und sind mit Standardsoftware nicht hinreichend abzubilden (Hornung et al. 2005). Möchte man mit den Primärsystemen einen strukturierten Behandlungsprozess innerhalb eines Ärzteverbundes unterstützen, z.B. eine angepasste Koordination und Vergütung von Facharztterminen oder Krankenhauseinweisungen, so müssen alle teilnehmenden Primärsysteme an den Prozess angepasst werden. Regionale Ärzteverbünde, die institutionsübergreifende administrative und medizinische Aufgaben koordinieren, die von den Praxen nicht eigenständig durchgeführt werden können, nutzen jedoch im Regelfall eine Vielzahl von proprietären AIS. Der regionale Ärzteverbund, in dem diese Forschungsarbeit durchgeführt wurde, ist ein Praxisnetz von 493 niedergelassenen Ärzten, in dem gegenwärtig 40 verschiedene AIS zum Einsatz kommen (Dünnebeil et al. 2011). Die Vielzahl der AIS macht eine Bereitstellung institutionsübergreifender Prozesse, die regionale Anforderungen ohne Medienbrüche implementieren, auf den Primärsystemen unmöglich, da sonst die Anpassung von bis zu 40 Systemen mit deren Herstellern koordiniert werden muss (Abb. 2).

\subsection{Mehrwertanwendungen der TI}

Aus den genannten Gründen wird, unabhängig von der Nutzung standardisierter Primärsysteme, die Entwicklung und Bereitstellung von MWA angestrebt, um regional integrierte Versorgungsprozesse zu implementieren. MWA stellen „eine „[...] verteilte Anwendung, bestehend [...] aus der Summe aller Schnittstellen, Protokolle und Komponenten, die für die Umsetzung eines fachlichen Use Cases notwendig sind" (gematik 2009a) dar. Die Anwendungen können, unter Nutzung der Dienste der eGK, bestehende Abläufe und Aufgaben vereinfachen oder neue Anforderungen implementieren, etwa in Praxisnetzen oder medizinischen Versorgungszentren (Dünnebeil et al. 2011). So erhalten medizinische Institutionen die Möglichkeit, eigene Anforderungen umzusetzen und auf die bestehenden Funktionalitäten der 
TI zurückzugreifen. Abbildung 3 zeigt schematisch eine MWA, die innerhalb eines Ärzteverbunds eine qualitätsgesicherte Überweisung elektronisch abbilden soll. Die erarbeitete Architektur soll eine strukturierte Entwicklung solcher Anwendungen unterstützen.

Ziel des vorliegenden Beitrags ist es, für eine Architektur für verteilte MWA der TI die Kompositionseinheiten, die in solchen Anwendungen bedeutend sind, $\mathrm{zu}$ identifizieren, zu abstrahieren und zu dokumentieren. So können MWA zukünftig in Anlehnung an eine strukturierte Architektur entworfen werden (Shaw und Garlan 1996). Einzelne Module können damit stringent modelliert, implementiert und wieder verwendet werden. So soll die Grundlage für die Weiterentwicklung hin zu einer Referenzarchitektur für MWA und langfristig zu einem Referenzmodell für dezentrale Versorgungsprozesse im ambulanten Gesundheitswesen gelegt werden.

\subsection{Softwarearchitekturen im Gesundheitswesen}

Nach Dern (2009) ist eine IS-Architektur die strukturierende Abstraktion existierender oder geplanter IS. Ziele von Softwarearchitekturen sind Dokumentation, Kommunikation, Wiederverwendung, Grundlage für den Systementwurf und Qualitätssicherung (Hasselbring 2000). Softwarearchitekturen beschreiben nach Perry und Wolf (1992) die Auswahl an Softwarekomponenten, deren Interaktionen und ihre Datenstrukturen, die sich aus Anforderungen ableiten und die Basis für den Softwareentwurf bilden. Eine Komponente in der
Softwarearchitektur ist nach Szyperski et al. (2002) eine Kompositionseinheit, die wohldefinierte Schnittstellen und explizite Zusammenhänge besitzt, und unabhängig von anderen eingesetzt oder mit anderen kombiniert werden kann. Die allgemeine Ausgestaltung von Komponenten und ihren Interaktionsmustern kann anhand domänenunabhängiger Architekturmuster vorgenommen werden. Schichtenbasierte Architekturmuster dienen der Strukturierung unklarer Anforderungen (Buschmann et al. 1996). Serviceorientierte Architekturmuster, wie sie in der TI vorgesehen wurden, ermöglichen durch Neukomposition von atomaren Services die flexible Umsetzung neuer Prozesse (Tsai et al. 2006). Refe- renzarchitekturen kombinieren nach Vogel et al. (2009) allgemeines Architekturwissen mit spezifischen Anforderungen zu einer architektonischen Gesamtlösung für einen Bereich, die als Grundlage für die Konzeption konkreter Architekturen dient. Referenzmodelle, die vornehmlich in der Wirtschaftsinformatik Verwendung finden, versuchen das gesamte Informationsmodell eines Anwendungsbereichs als Referenz für Unternehmensmodelle herauszuarbeiten (Fettke und Loos 2002).

Dinh und Chu (2006) klassifizieren den Gesundheitssektor als eine der komplexesten und informationsintensivsten Umgebungen. Als Gründe werden unter anderem die Komplexität der Prozes-

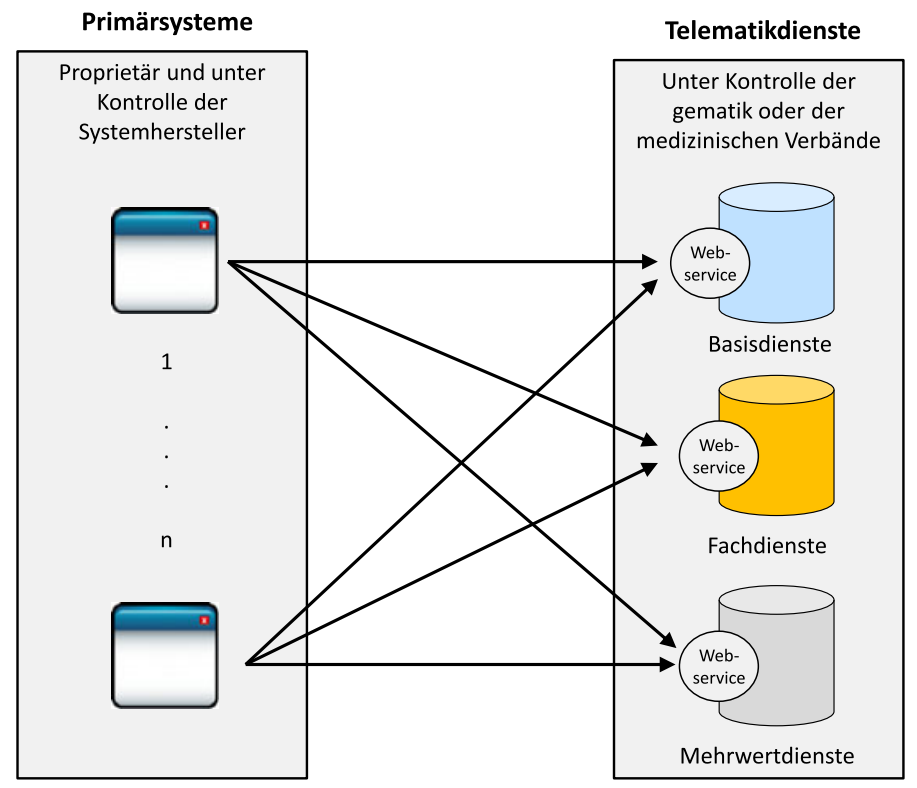

Abb. 2 Die SOA der deutschen Gesundheitsversorgung

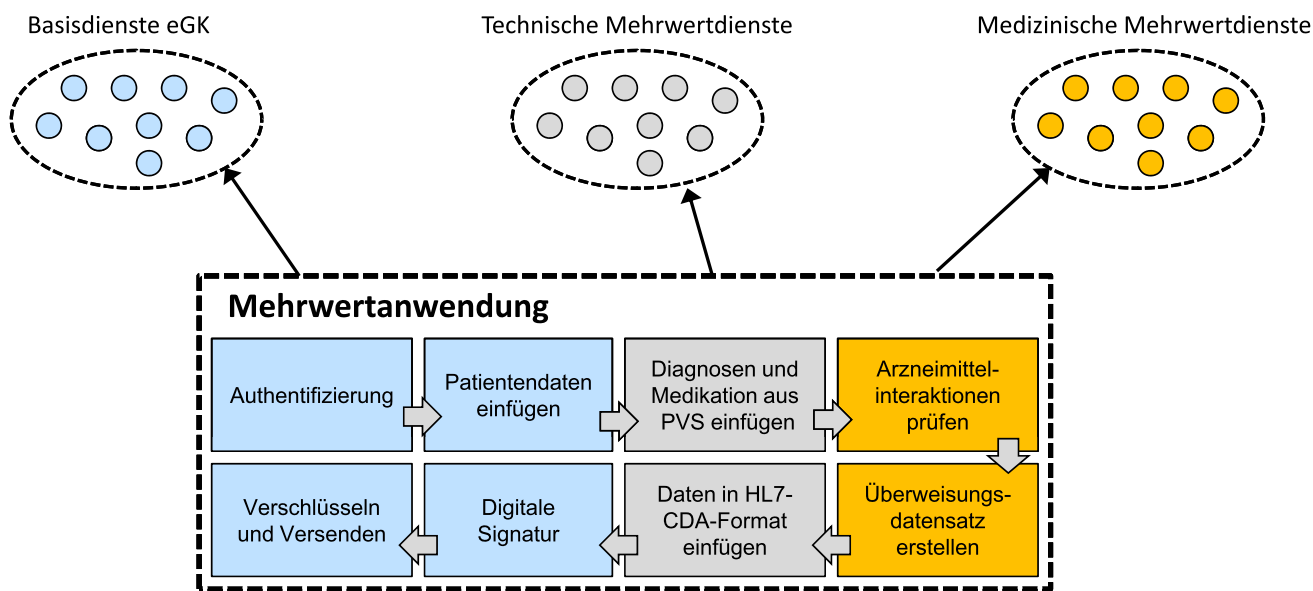

Abb. 3 Schematische Darstellung einer MWA zur eÜberweisung 
se (speziell von klinischen Pfaden), die große Anzahl an klinischen Datenformaten (z.B. für Diagnostik, Befunde oder klinische Bildgebung), die Mehrdeutigkeit klinischer Dokumentationskonzepte, die hohe Variabilität medizinischer Akten oder die heterogenen Organisationsstrukturen in medizinischen Institutionen genannt. Bisherige Arbeiten strukturieren E-Health-Architekturen primär nach den Gesichtspunkten von Interoperabilität (Pedersen und Hasselbring 2004), Integrationsfähigkeit (Tsiknakis et al. 2002; Schweiger et al. 2007), Sicherheit (Blobel 2004; Gritzalis und Lambrinoudakis 2004; Riedl et al. 2008) oder fachspezifischen Gesichtspunkten (Fayn et al. 2003). Eine Architektur für Telematikdienste wurde in Tsiknakis et al. (1997) nach technischen Gesichtspunkten in Schichten gruppiert und in Fraunhofer (2005) als SOA spezifiziert. Letztere folgt einer Schichtenarchitektur aus Sicherheitsgesichtspunkten. Eine Architektur für ausgewählte Fachanwendungen der TI wurde zwar grob skizziert (Frießem et al. 2005), jedoch nicht hinreichend ausgearbeitet, um für heterogene MWA einsetzbar zu sein. Die in diesem Beitrag beschriebene Architektur für Anwendungen der TI soll - in Anlehnung an bestehende E-Health Architekturen - in Schichten aufgebaut werden (Dijkstra 1968), da sich ein solcher Ansatz in vielen Internetanwendungen bewährt hat (Urgaonkar et al. 2007). Auch das erfolgreiche ISO/OSI-Referenzmodell (Zimmermann 1980) wird zum Vorbild genommen, um verschiedene Kommunikationsaufgaben bei der sicheren Kommunikation von verteilten Systemen im medizinischen Bereich abzubilden. Die Nutzung von Diensten in einer SOA kann nach funktionalen Kriterien modularisiert werden, um eine strukturierte Anwendung zu modellieren (Arsanjani und Allam 2006). Bei der Verwendung des serviceorientierten Architekturmusters, speziell für verteilte Groupware, die, wie die TI, XML-basierte Daten verarbeitet, hat sich die Form der Komposition bewährt (Marsic 2001). Wie Dienste, die meist atomare Funktionalitäten anbieten sollen, in einer erweiterten SOA kombiniert werden können, wurde in $\mathrm{Pa}$ pazoglou (2003) gezeigt. Die Architektur für MWA der TI zielt daher auf eine Gliederung der Applikationen nach funktionalen Schichten, die aus Anforderungen folgen, die bei den Nutzern erhoben werden.

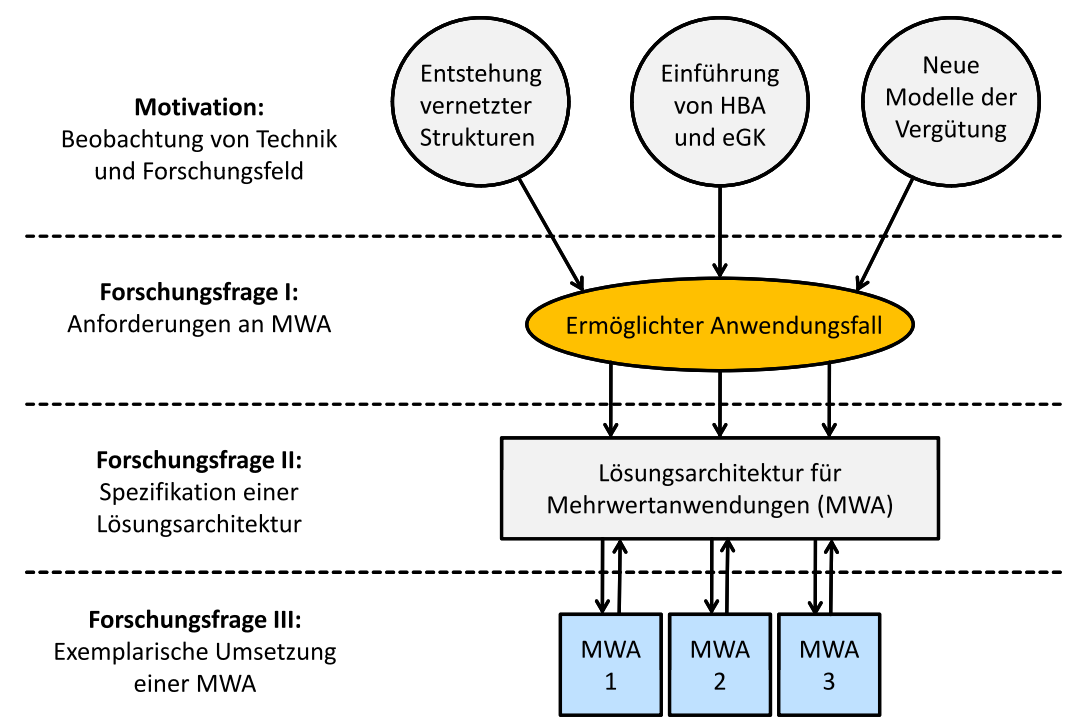

Abb. 4 Forschungsmethode

\section{Forschungsmethodik}

Der Beitrag verwendet einen gestaltungsorientierten Ansatz nach Hevner et al. (2004). Das Artefakt, welches im Zentrum der Betrachtung steht, ist eine Softwarearchitektur für die Implementierung telematikbasierter MWA im deutschen Gesundheitswesen. Um die Architektur aus den Anforderungen der Nutzer abzuleiten, wurde eine repräsentative quantitative Befragung von 117 der 500 niedergelassenen Ärzte im Praxisnetz GO IN e.V. (Dünnebeil et al. 2010a; Dünnebeil et al. 2010b; Dünnebeil et al. 2012) durchgeführt. Ferner wurden qualitative Interviews mit jeweils zwei Hausärzten, zwei Fachärzten, den Vorständen des Praxisnetzes GO IN e. V. und einer Krankenkasse geführt. Die Krankenkasse und das Praxisnetz haben seit Oktober 2011 einen Integrierten Versorgungsvertrag geschlossen, um die Versorgung der Region nach leistungsbezogenen Kriterien zu verbessern (Audi BKK 2011). In der Befragung wurden übergreifende Anforderungen an MWA in offenen Fragen erfasst, die die Basis für die zu entwickelnde Architektur bilden, und fachliche Anwendungsfälle mit Nutzenpotenzialen identifizieren. In Anlehnung an die Rahmenbedingungen, die in Kapitel 1 beschrieben wurden, wird in der Folge ein identifizierter Anwendungsfall, der, durch die Verfügbarkeit der TI, die fortschreitende Kooperation in Ärzteverbünden und neue Vergütungsmechanismen auf Basis von $\$ 140$ SGB5 ermöglicht, beschrieben und implementiert (Abb. 4).
Für den vorliegenden Beitrag wurde die elektronische Koordination von Terminen und Überweisungen für Facharztbesuche (eÜberweisung) als Beispielanwendung gewählt. Der theoretische Beitrag der Architektur ist nach (Gregor 2006) eine Theorie für das Design eines medizinischen IS. Die Architektur reiht sich damit in die Beiträge zum Softwareengineering ein, die in diesem Zusammenhang Systementwicklungsansätze untersuchen. Die Verifikation der Architektur der eÜberweisung stellt einen Prototyp einer telematikbasierten MWA dar, der Anforderungen von Ärzten auf Basis der technischen Infrastruktur der TI umsetzt, wie es in Hevner u. a. (2004) gefordert wird.

\section{Ergebnisse der Architekturspezifikation}

\section{1 Übergreifende Anforderungen}

Aus den durchgeführten empirischen Erhebungen, den qualitativen Befragungen der Leistungserbringer und einer Literaturanalyse wurden übergreifende Anforderungen abgeleitet, die für MWA von herausragender Bedeutung sind. Tabelle 1 führt zehn wichtige Anforderungen auf, die in der Architektur abgedeckt werden sollen. Die Anforderungen werden kurz begründet und in den nachfolgend spezifizierten Architekturkomponenten erläutert. 
Tab. 1 Übergreifende Anforderungen (ÜA)

$\ddot{U A} 1$

Auf die dauerhafte zentrale Speicherung von personenbezogenen medizinischen Daten soll in MWA verzichtet werden.

Die Ärzte im Praxisnetz (53 \%) sprechen sich klar gegen die zentrale Speicherung von Patientendaten aus und bestätigen damit eine Reihe gleich lautender Voruntersuchungen (TKK 2009; Allensbach 2010).

$\ddot{U A} 2$

MWA sollen sich, wenn möglich, an etablierten Versorgungsprozessen orientieren, um bestehende Anwendungen und Abläufe der Leistungserbringer einbinden zu können.

So soll der verbreiteten Angst vor Überforderung durch neue IT-gestützte Aktivitäten entgegengewirkt werden, um die Akzeptanz von MWA durch Verbesserung eingespielter Prozesse zu steigern.

Der in MWA abgebildete Versorgungsprozess muss den bestehenden Prozess ersetzen können. Eine parallele Ausführung ist nicht hinnehmbar.

Digitale Überweisungen, Verordnungen, Einweisungen oder Datenübermittlungen sehen die Ärzte nur als realistisch an, wenn diese nicht zusätzlich zu den papierbasierten Aktivitäten durchgeführt werden müssen.

Interoperabilität zur Vermeidung redundanter Datenerfassung ist notwendig, um manuelles Übertragen von Daten in die Primärsysteme zu vermeiden.

Für die manuelle Erfassung von Fremdbefunden oder Laborergebnissen bleibt im Praxisalltag keine Zeit. Daten aus anderen Systemen werden gegenwärtig oft nur gedruckt und der Papierakte beigelegt.

Die Möglichkeit, fall- und netzspezifische Anforderungen unabhängig von den Herstellern der Primärsysteme durch MWA abzubilden, muss gegeben sein.

Die Fokusgruppen der Haus-, Fach- und Krankenhausärzte äußerten den Wunsch, eigenständig zu definieren, welche Daten im Rahmen eines Versorgungsprozesses erhoben und übermittelt werden sollen.

Erstellung und Bearbeitung von medizinischen Datenobjekten muss eindeutig einer Person zugeordnet werden können, die ausreichende Autorisierung besitzt.

Nachvollziehbarkeit und Rechtssicherheit sind zentrale Bedenken bei der Verwendung medizinischer Bestandsdokumentation. Der Wunsch nach Transparenz und persönlicher Zurechenbarkeit wurde hier berücksichtigt.

MWA müssen auf etablierten und zertifizierten Sicherheitskonzepten aufgebaut werden.

Diese Anforderung entspricht dem Wunsch einer Zertifizierung von MWA durch den Bundesdatenschützer.

$\ddot{U} A 8$ Praxismitarbeiter delegieren können.

Hier wurden die Wahrung der Patientenrechte (\$291a SGB5), die Entwicklung patientenzentrierten Gesundheitswesens (Stewart et al. 2000) und die Gleichbehandlung von IT-Nutzern und anderen Patienten betont.

Medizinische und administrative Aufgaben müssen möglichst getrennt werden können.

Die Ärzte waren offen für zusätzliche Aktivitäten zur Qualitätssicherung oder Effizienzsteigerung. Sie möchten Aufgaben, die keine medizinischen Kompetenzen erfordern, jedoch an Helfer delegieren.

Die Möglichkeit zur Erfassung und Auswertung von wohldefinierten Steuerungsdaten aus den Versorgungsprozessen muss gegeben sein.

Das Praxisnetz möchte für die Vergütung und Qualitätssicherung bspw. erfassen, bei welchen Diagnosen Einweisungen, Verordnungen und Überweisungen angestoßen und wie diese eingelöst wurden.

3.2 Ableitung einer Architektur für MWA der TI

Nach ÜA2 sollen MWA bestehende Prozesse verbessern (Frießem et al. 2005). Ziel ist die Verwirklichung eines strukturierten, durchgehenden Datenflusses über Institutsionengrenzen hinweg, der in Versorgungsprozesse eingebunden ist. Als Ausgangspunkt wurden die etablierten Prozesse zur Verordnung, zur Überweisung und zum Informationsaustausch im Gesundheitswesen studiert und verbessert. Abbildung 5 illustriert, wie der Prozess der Überweisung (vgl. Anhang 3; online verfügbar über http://springerlink.com) in Funktionseinheiten und -ebenen aufgegliedert wurde, um den Nachrichtenfluss zwischen Institutionen zu implementieren.

Um eine zentrale Speicherung von $\mathrm{Pa}$ tientendaten zu verhindern (Dünnebeil et al. 2010b), werden die Daten auf lokalen Systemen in den jeweiligen Institutionen gespeichert und über eine administrative Funktionseinheit verknüpft (ÜA1). Im zweiten Schritt der Architekturspezifikation wurden nach ÜA9 die administrativen Aufgaben von den Medizinischen in zwei MWA getrennt (Rohner und Winter 2008). Die medizinische MWA wird mit den Primärsyste- men verbunden, um den Austausch der Daten zu ermöglichen (ÜA4). Die Vermittlung der Patientendaten wird in Anlehnung an \$291a SGB5 durch die Patienten selber organisiert (BRD 2004a). Für Patienten mit Einschränkungen bezüglich der Systemnutzung besteht die Möglichkeit, diese administrativen Aufgaben an medizinisches Hilfspersonal zu delegieren (ÜA8). Im dritten Schritt werden die administrativen und medizinischen Aufgaben in Funktionsebenen aufgegliedert, die wohldefinierte Aufgaben aus den übergreifenden Anforderungen abdecken. Die Funktionsebenen bilden die Grundlage für ein Softwaremodul mit 


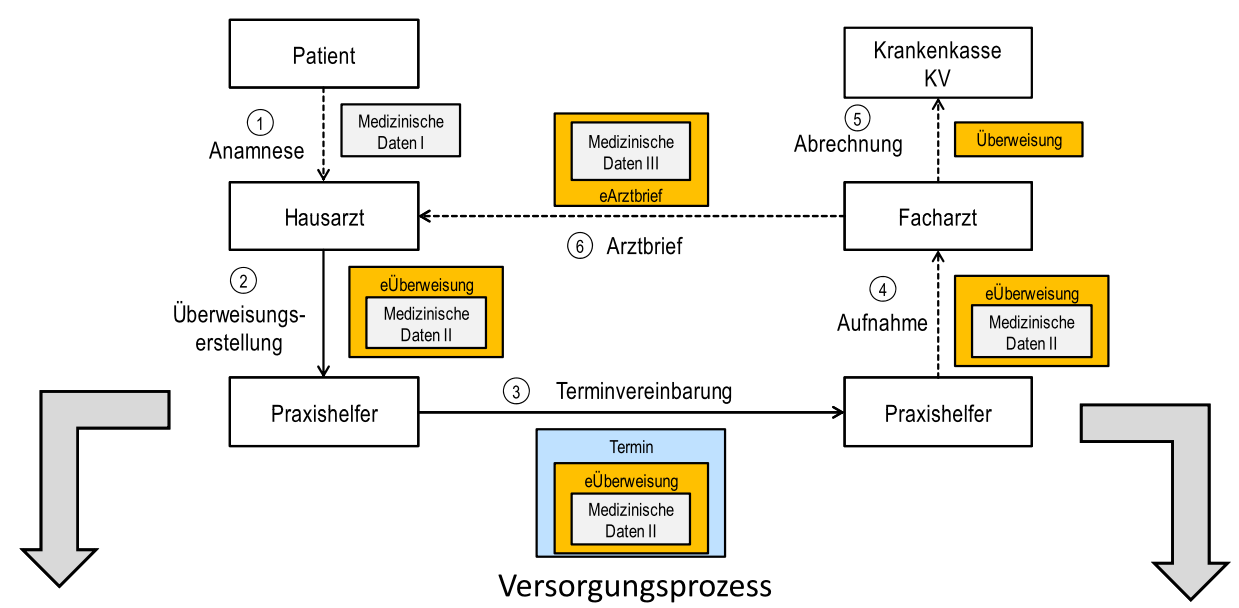

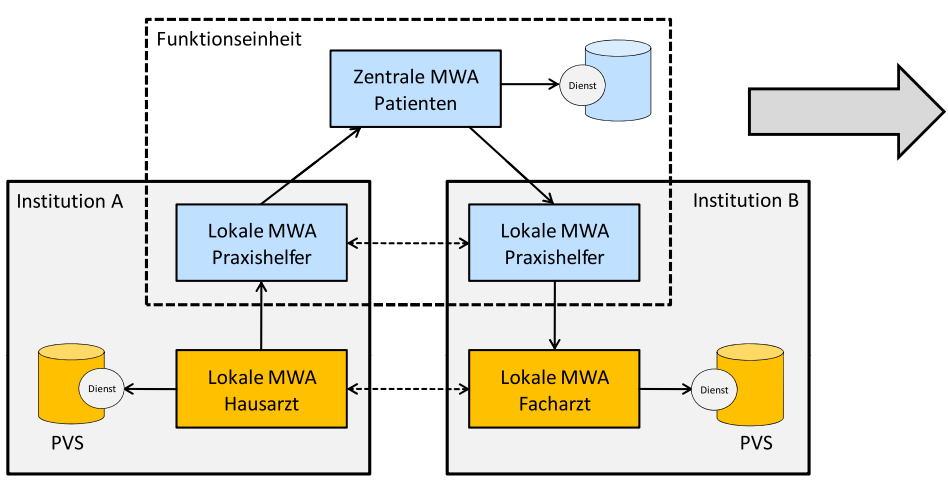

Funktionseinheiten der Stakeholder

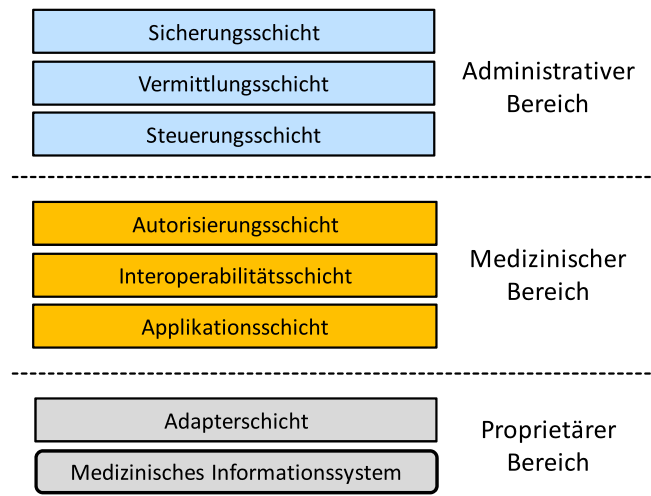

Funktionsebenen

Abb. 5 Strukturierte Ableitung der e-Health Architektur

hoher Kohäsion. So wird die Wiederverwendbarkeit und Standardisierung von Komponenten im Gesundheitswesen erleichtert. Die Ebenen sind den bestehenden Aufgaben von Arbeitsabläufen im Gesundheitswesen nachempfunden und bilden deren digitales Komplement. Der Drucker bildet heutzutage einen Adapter, der medizinische Daten aus den Primärsystemen auf ein Formular druckt. Aktuell wird die Interoperabilität im $\mathrm{Ge}$ sundheitswesen durch die Spezifikation von Formularen durch übergeordnete Institutionen ermöglicht (KBV 2011b) und die Unterschrift eines Vertragsarztes stellt die hinreichende Autorisierung des Ausstellers sicher. Die Vermittlung wird gegenwärtig bei unbekannten Adressaten (bspw. Fachärzten oder Apotheken) persönlich durch Patienten, bei bekannten Adressaten durch Post oder Fax vorgenommen. Die Sicherungs- (ÜA7) und Steuerungsschicht (ÜA10) folgen direkt aus den Anforderungen. Die Applikationsschicht ermöglicht eine innovative Verwaltung und Aufbereitung medizinischer Daten auf Seite der Ärzte. Die
Ebenen werden nachfolgend im Detail beschrieben.

\subsubsection{Adapterschicht}

Auf dieser Schicht wird die Frage beantwortet, welche Primärsysteme eingebunden werden sollen und wie die Einbindung realisiert werden kann. Für Ärzte ist die automatisierte Verarbeitung ihrer Daten ein essenzielles Kriterium (Dünnebeil et al. 2010a). Die doppelte Erfassung von medizinischen Daten in Primärsystemen und in MWA wird ausdrücklich abgelehnt, da sonst redundante Daten erhoben werden müssen (ÜA4). Die Arbeit mit den Primärsystemen ist für die Mehrzahl der Leistungserbringer dennoch verbindlich, um ihre Leistungen bei den Kostenträgern abrechnen zu können. Die Adapterschicht stellt die Verbindung zwischen den IS von Leistungserbringern oder Patienten und den MWA her. Während Primärsysteme meist proprietäre und damit geschlossene Systeme sind, können MWA an die regiona- len Versorgungsprozesse angepasst werden. Primärsysteme dienen entweder der Erhebung von medizinischen Daten, wie etwa ein Gerät aus der Medizintechnik, oder zur Behandlungsdokumentation und Abrechnung, wie ein AIS. Sie stellen persistente Datensilos mit verteilten Patientendaten dar, wenn keine übergreifenden Patientenakten vorhanden sind. Der Adapter bietet nach außen einheitliche Schnittstellen, die von verschiedenen MWA verwendet werden können. Je nach Anwendungsfall müssen Daten aus einem Primärsystem gelesen oder in die Primärsysteme der Zielinstitution geschrieben werden. Dazu greift der Adapter entweder auf die Schnittstellen bzw. die Datenbank der Primärsysteme zu und kapselt die proprietäre Datenquellen hinter einer standardisierten Schnittstelle. Diese können bspw. als Webservice bereitgestellt werden, um die Integration der Primärsysteme nach dem SOAParadigma zu gewährleisten (Mauro et al. 2009). 


\subsubsection{Applikationsschicht}

Auf dieser Schicht wird die eigentliche medizinische Anwendung umgesetzt, die den Mehrwert bereitstellt. Solche Anwendungen bieten Funktionen, die nicht von den Primärsystemen der Leistungserbringer angeboten werden. Hier wird einer breiten Entwicklergemeinde die Möglichkeit eröffnet, Innovationen in das Gesundheitswesen zu tragen. Entwickler sind somit nicht mehr abhängig von der Offenheit und den Lebenszyklen der Primärsysteme, und Verbesserungen der Behandlungsqualität durch IT-Innovationen bleiben nicht auf die Hersteller weniger Primärsysteme beschränkt. Positive Entwicklungen durch den Einsatz von IT-Systemen in der Behandlungsunterstützung wurden in vielen Studien belegt (Sarv und Rajiv 2000; Sunyaev und Chornyi 2012; Wu et al. 2006). Durch einen Abgleich von Arzneimittelwechselwirkungen konnte bspw. die Anzahl von Fehlbehandlungen drastisch gesenkt werden (Lappé et al. 2004). Die fallbezogene Selektion von medizinischen Daten für einen weiterbehandelnden Arzt kann bei Krankenhauseinweisungen die Anzahl von Doppeluntersuchungen reduzieren (Sawicki 2005).

\subsubsection{Interoperabilitätsschicht}

Auf dieser Schicht muss die Entscheidung getroffen werden, wie verteilte MWA in die Lage versetzt werden sollen, miteinander $\mathrm{zu}$ interagieren. Interoperabilität bezeichnet die Fähigkeit von autonomen Anwendungen unterschiedlicher Herkunft und heterogener Machart, miteinander kommunizieren zu können (Wegner 1996). Es wird in der Regel zwischen syntaktischer Interoperabilität, die die Datenübertragung zum Zielsystem und die dortige Verarbeitung implementiert und gewährleistet, und semantischer Interoperabilität, die Bedeutung und Verwendung der Daten regelt, unterschieden (Ouksel und Sheth 1999). Ohne zentrale Speicherung von Daten und deren Nutzung über eine einheitliche Benutzerschnittstelle muss ein einheitliches Austauschformat zwischen Sender und Empfänger definiert werden. Das System des Senders überführt die Daten in das vorgeschriebene Austauschformat, der Empfänger extrahiert sie aus diesem. Das Thema Interoperabilität ist ein breites Forschungsfeld in der medizinischen Informatik (Pedersen und Hasselbring 2004;
Eichelberg et al. 2005). Es wurden bereits interoperable Austauschformate wie $\mathrm{He}$ alth Level 7 (HL7) Clinical Document Architecture (CDA) und der Continuity of Care Record (CCR) spezifiziert (Ferranti et al. 2006). So können Daten in das IS des Empfängers eingefügt werden, ohne dass eine redundante Erfassung notwendig wird. Mögliche Übertragungsfehler und Zeitaufwände beim erneuten manuellen Erfassen der Daten von Formularen können reduziert werden. Im Gesundheitswesen existieren verschiedene Kommunikations- und Dokumentationsstandards sowie übergreifende medizinische Begriffssysteme, die Interoperabilität ermöglichen (Pedersen und Hasselbring 2004). Bei übergreifenden Standards ist die Überführung in die Primärsysteme der Zielinstitution erheblich einfacher, da keine speziell angepasste Adapterschicht notwendig ist.

\subsubsection{Autorisierungsschicht}

Auf dieser Schicht ist die Frage zu klären, welcher Personenkreis autorisiert wird, Transaktionen in der MWA abzuwickeln, und wie dieser Mechanismus technisch umzusetzen ist (ÜA6). Dafür wurden in der Informatik bereits weitreichende Konzepte für das Gesundheitswesen entwickelt (Chandramouli 2001). In medizinischen Prozessen entstehen oft wechselseitige Verbindlichkeiten. Weiterbehandelnde Ärzte müssen der Behandlungsdokumentation des Vorgängers vertrauen können, wenn sie diese als Entscheidungsgrundlage verwenden, ohne eine Doppeluntersuchung durchzuführen. Auch Apotheken müssen der Echtheit einer Verordnung vertrauen können und sicherstellen, dass der Aussteller zur Verordnung des Präparats befähigt war. Heute wird dies in der Regel durch Institutionsstempel und Unterschrift eines Vertragsarztes bestätigt. Für digitale Medien kann z. B. eine elektronische Signatur verwendet werden (Rivest et al. 1978). Da die Möglichkeit für zwischenmenschliche Vertrauensbildung oft nicht besteht (Kumbruck 2000), müssen geeignete technische Möglichkeiten bereitgestellt werden, um Signaturen zu verifizieren und einer Gruppe von Leistungserbringern zuzuordnen. Hierfür muss eine Institution benannt werden, die entsprechende Zertifikate zur Verfügung stellt und verwaltet. Diese kann auch Passwörter, persönliche Identifikationsnummern oder Transaktionsnummern (TAN) zur Verfügung stellen. Rechtsverbindliche Transaktionen oder Dokumente, wie Verordnungen, bedürfen oft einer Qualifizierten Digitalen Signatur, während andere Dokumente, wie etwa Arztbriefe, auch ohne Signatur versendet werden dürfen.

\subsubsection{Steuerungsschicht}

Auf dieser Schicht muss zur Gewährleistung von ÜA10 die Möglichkeit umgesetzt werden, Steuerungsdaten im Rahmen von Versorgungsprozessen zu erheben und auszuwerten. Zur Abrechnung von Leistungen aus integrierten Versorgungsverträgen nach $\$ 140$ SGB5 kann von den Vertragspartnern erfasst werden, welche Leistungen von einem Arzt erbracht worden sind, um diese außerhalb des Regelleistungsvolumens zu vergüten. So entsteht zu jedem Versorgungsprozess ein wohldefinierter Datensatz, der zur Steuerung und Qualitätssicherung auf Managementebene genutzt werden kann. Diese Daten können auch zur Steigerung der medizinischen Qualität der Behandlungen im Ärzteverbund dienen, indem Ärzten Empfehlungen aus der evidenzbasierten Medizin an die Hand gegeben werden, die auf Realdaten basieren (Sackett et al. 1996). Patientendaten können zu diesem Zweck in Data Warehouses aufbereitet (Stolba und Tjoa 2006) und hinreichend anonymisiert und aggregiert werden, um die Erfüllung von ÜA1 zu gewährleisten (Sweeney 1997). Auch die Bereitstellung von Netzdaten kann für einen Versorgungsprozess von Bedeutung sein. Eine Auswahl bevorzugter Lieferanten oder zeitnaher Behandlungstermine kann zusätzlich zum medizinischen Dokument zur Verfügung gestellt werden, um die spätere Vermittlung der Dokumente an einen weiteren Leistungserbringer nach ökonomischen oder organisatorischen Gesichtspunkten $\mathrm{zu}$ steuern. Eine willkürliche und umfassende Sammlung und Auswertung der Daten aus MWA, wie es in Mobilfunkoder Internetanwendungen häufig geschieht (O'Brien 2010), muss unterbunden werden. Der Entwurf der Steuerungsdatenschicht muss einem strukturierten Ansatz zum Privacy-Engineering folgen (Kaletsch und Sunyaev 2011).

\subsubsection{Vermittlungsschicht}

Auf dieser Schicht muss die Frage geklärt werden, an welche Empfänger Daten vermittelt werden sollen und wie die- 
se Vermittlung technisch umgesetzt werden soll. Gegenwärtig bekommen die Patienten eine Verordnung oder einen Arztbrief ausgehändigt und übernehmen anschließend die Vermittlung der Dokumente eigenständig. So kann etwa eine Verordnung von Medikamenten in der nächstgelegenen Apotheke eingelöst werden, ein Facharzttermin oder eine Taxifahrt zum Facharzt über das Telefon vereinbart werden. Die digitale Vermittlung von medizinischen Daten, z.B. elektronische Verordnung von Medikamenten oder orthopädischen Hilfsmitteln, kann hingegen nach festgelegten Regeln vonstattengehen, die im Vorhinein aufgrund von ökonomischen oder medizinischen Gesichtspunkten festgelegt wurden. Die Vermittlung folgt damit objektiven Regeln. Die Voraussetzung für diese $\mathrm{Ob}$ jektivierung der Vermittlung kann vorher auf der Steuerungsschicht gewährleistet werden. So können mit Hilfe von Netzinformationen, die etwa die günstigsten Preise oder die besten Lieferkonditionen für ein Medikament feststellen, gezielte Vermittlungen vorgenommen werden. Hat ein Arzt die Verordnung eines Hilfsmittels ausgestellt, kann diese bei dem Sanitätshaus eingelöst werden, das den günstigsten Preis, die schnellste Lieferung oder die beste Qualität garantiert. Auch die Terminvergabe kann nach objektiven Regeln geregelt werden. So kann automatisch der nächstmögliche Facharzttermin reserviert werden, bei dem die Praxis innerhalb eines Radius von $10 \mathrm{~km}$ liegt, der Arzt einem bestimmten Versorgungsvertrag beigetreten ist und der Arzt eine gute Bewertung hat.

\subsubsection{Sicherungsschicht}

Datensicherheit und Vertraulichkeit ist im Bereich medizinischer Daten ein Aspekt mit herausragender Bedeutung (Rindfleisch 1997; Stanford 2002; Sunyaev und Pflug 2012). Vor diesem Hintergrund muss die Frage beantwortet werden, welche Sicherheitsstandards verwendet werden und welche Mechanismen für die Umsetzung gewählt werden. Sowohl Befürworter als auch Gegner der Telematik sprechen sich mit großer Mehrheit für die Verwendung von verbindlichen und übergreifenden Sicherheitsstandards in Telematikanwendungen aus (Dünnebeil et al. 2010a). Auch der deutsche Gesetzgeber regelt die $\mathrm{Zu}$ griffsberechtigung, die Auditierung und die Verschlüsselung von Telematikanwendungen explizit im Sozialgesetzbuch
(BRD 2004a). Der amerikanische „Health Insurance Portability and Accountability Act (HIPAA)“ stellt darüber hinaus genaue gesetzliche Regeln für den Umgang mit digitalen Gesundheitsdaten auf, die neben der administrativen und physischen Sicherheit von Gesundheitsdaten auch die technischen Aspekte regeln (Mercuri 2004). Verstöße gegen die HIPAA-Richtlinien werden mit hohen Geld- und Gefängnisstrafen geahndet. Für MWA der TI müssen sich die Verantwortlichen auf ein gesetzeskonformes Sicherheitsniveau einigen, das ein ausgewogenes Verhältnis zwischen Nutzbarkeit und Schutzbedarf findet, und es auf der Sicherungsschicht mit geeigneten Mechanismen umsetzen.

\section{Beispielhafte Umsetzung einer MWA}

Zur Illustration der Architektur wurde eine konkrete MWA umgesetzt, die ein Anwendungsszenario unterstützt. Auf Basis der abstrakten Architektur wurde in diesem Rahmen eine konkrete Facharchitektur spezifiziert. In den Gesprächen mit den eingebundenen Praxispartnern wurde die eÜberweisung als ein Anwendungsfall mit hohem Nutzen identifiziert.

\subsection{Funktionale Anforderungen}

Eine Facharztüberweisung dient im deutschen Gesundheitswesen dazu, Patienten die Berechtigung zu einem Facharztbesuch zu erteilen. Diese wird durch einen Hausarzt mit Kassenzulassung erteilt. Im traditionellen Prozess stellt das Überweisungsformular eine ungerichtete Berechtigung zur Abrechnung einer Facharztbehandlung dar, die nach der Vereinbarung eines Facharzttermins durch den Patienten an die Facharztpraxis übergeben wird. Patienten wählen durch Terminvereinbarung implizit einen Empfänger, dem sie die medizinische Dokumentation zur Verfügung stellen. So wird aus der ungerichteten Kommunikation eine gerichtete. Die hier beschriebene MWA zielt darauf, den Prozess der Terminvereinbarung mit dem der Überweisung und der Kommunikation medizinischer Inhalte $\mathrm{zu}$ verbinden. So werden traditionelle Prozesse kombiniert, digitalisiert, angereichert und vereinfacht. Dafür werden die Prozesse der Überweisung und der Terminvereinbarung kombiniert. Der
Mehrwert der Applikation liegt in einer digitalen Autorisierung der Patienten, dem integrierten elektronischen Terminmanagement und der fachbezogenen Selektion und Übermittlung digitaler medizinischer Daten.

Das Angebot der Reservierung von Facharztterminen über eine zentrale Plattform zielt auf die Entlastung von Patienten und Arzthelfern in den Praxen, da die Vereinbarung zeitnaher Facharzttermine oft mit erheblichem Kommunikationsaufwand verbunden ist. Das Auffinden von zeitlich oder räumlich günstig gelegenen Terminen soll durch adäquate Darstellung im System vereinfacht werden. Die eÜberweisung soll von Hausärzten erstellt und durch eine qualifizierte elektronische Signatur rechtskräftig werden. Hausärzten wird ferner die Möglichkeit geboten, medizinische Daten aus ihren lokalen AIS in das digitale Überweisungsformular zu überführen, ohne diese manuell eintragen zu müssen, um sie zur automatisierten Weiterverwendung an den Facharzt zu übermitteln. Funktionale Anforderungen, die in der Konzeptionsphase erhoben wurden, sind in Anhang 2 (verfügbar online über http://springerlink.com) aufgeführt. Die Schichten der Architektur für MWA wurden im Rahmen des Softwareentwurfs der eÜberweisung instanziiert. Zu diesem Zweck wurden die jeweiligen Komponenten spezifiziert und implementiert, um die Konzepttauglichkeit zu illustrieren. Abbildung 6 zeigt die Facharchitektur der eÜberweisung.

\subsection{Adapter für das Arzt-Informationssystem}

Die AIS-Adapterschicht stellt im Fall der eÜberweisung den Zugriff auf die Quellsysteme der Hausärzte und die Zielsysteme der Fachärzte sicher. Daten, die in die MWA überführt werden, können über die Schnittstellen der Anwendungskomponente aus den Primärsystemen abgerufen werden. Um die Daten aus den Primärsystemen, die gegenwärtig oft unstrukturiert vorliegen, maschinenlesbar aufzubereiten, müssen diese syntaktisch und semantisch kodiert werden, um Mehrdeutigkeiten auszuschlieBen. Zur Standardisierung wurden für Diagnosen das Terminologiesystem International Classification of Diseases Version 10 (ICD-10) und für Medikamente die Pharmazentralnummer (PZN) verwendet. Der Adapter wurde als Webservice umgesetzt, um einen einheitlichen 
Zugriff auf die Systeme zu gewährleisten. Abbildung 7 illustriert den Zugriff auf die Daten des Quellsystems und die Antwort des XML-Webservices. Probleme verursachen in dieser Komponente die heterogene Kodierung und Speicherung von medizinischen Daten in den Primärsystemen. Der Zugang zu den Daten erschließt sich nur nach eingehender Analyse der Datenhaltung. Die Überführung der oft rein textuell kodierten Attribute in übergreifende Terminologiesysteme gestaltet sich schwierig, da eine automatisierte Zuordnung oft nicht möglich ist. So konnten Laborbefunde nur im Originaltext ausgelesen werden; eine Aufbereitung der Maßeinheiten und Werte war aufgrund der Datenlage nicht möglich.

\section{3 Überweisungserstellung}

Die Komponente zur Überweisungserstellung greift über den Adapter auf Daten der Primärsysteme und über einen Konnektor auf Daten der eGK zu. Die Überweisungserstellung erlaubt es Ärzten, Daten fallbezogen auszuwählen, zu sortieren und für den Empfänger anzureichern. Die Facharztüberweisung enthält bisher medizinische Informationen, die vom Hausarzt auf das Formular gedruckt oder geschrieben werden können. Während die papierbasierte Überweisung nicht maschinenlesbar und auf die Größe DIN A5 beschränkt ist, können in einer eÜberweisung die Vorteile elektronischer Datenverarbeitung genutzt werden. Die Felder der eÜberweisung bilden eine beliebige Übermenge der derzeit verwendeten Papierformulare. Die Selektion der medizinischen Daten nach fallbezogenen Kriterien kann so mit beliebigen digitalen Elementen versehen werden, um die Krankengeschichte des Patienten adäquat wiederzugeben und weiterzuleiten (Shekelle et al. 2006). Es wurde eine Webanwendung in ASP.NET programmiert, die Nutzeroberfläche wurde an die Papierformulare angelehnt und im Rahmen der Anforderungen des Ärzteverbunds angepasst. Es wurden Hilfsmittel zur Auswahl von Diagnosen und Medikationen, wie Autovervollständigung und Vorlagen, implementiert. Eine Arzneimittelinteraktionsprüfung der überführten Daten über einen externen Webservice anhand der PZN wurde erprobt. Zentrale Schulungen für alle Nutzer im Praxisnetz sowie einfache Anpassung und Wartung sind aufgrund der Vereinheitlichung der Software

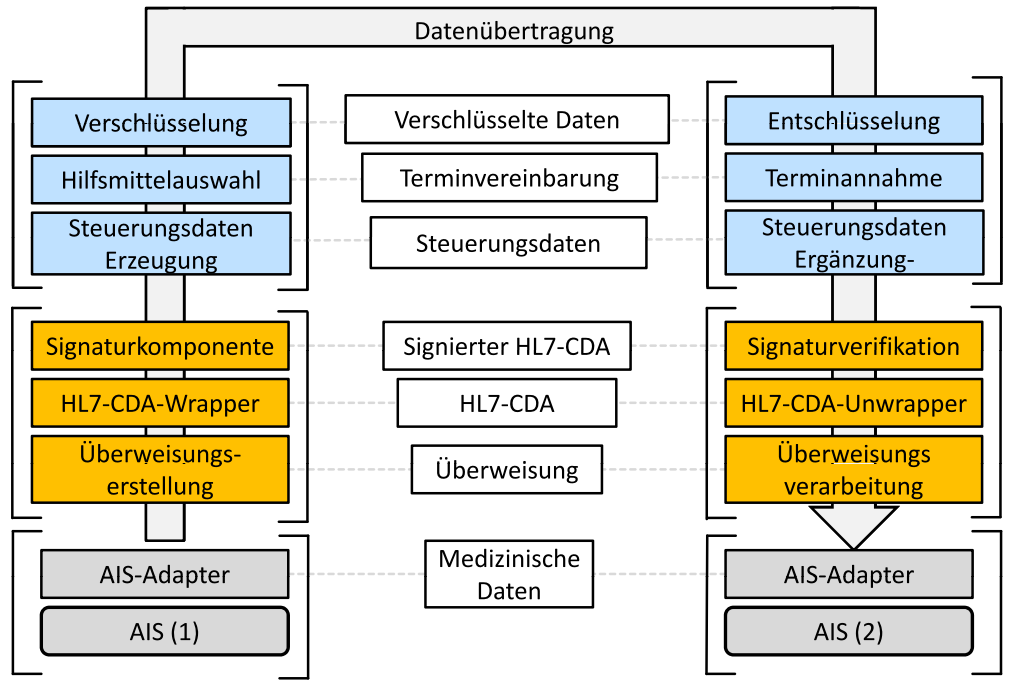

Abb. 6 Facharchitektur der eÜberweisung

\begin{tabular}{|c|c|c|c|}
\hline CBBSSPARTE & TMODIFY & MDOKUMENTA & CPATIENTENNR \\
\hline $\mathrm{D}$ & 20.10 .2010 & Ausschluss von Demenz $(\mathrm{F} 03+\mathrm{A})$ & 1013146301 \\
\hline $\mathrm{D}$ & 04.04 .2011 & Diabetisches Fußsyndrom (E14.74+G) & 1013146301 \\
\hline \multicolumn{4}{|c|}{$<$ ?xml version=" $1.0 "$ encoding="UTF- 8 "? $>$} \\
\hline \multicolumn{4}{|c|}{ - $<$ Diagnosen codeSystemName="ICD10gm2006" codeSystem="1.2.276.0.76.5.311" } \\
\hline \multicolumn{4}{|c|}{ languageCode="de-DE" version="TUM_D_V001"> } \\
\hline \multicolumn{4}{|l|}{$-<$ Diagnose $>$} \\
\hline \multicolumn{4}{|c|}{$<$ Datum $>20110404</$ Datum $>$} \\
\hline \multicolumn{4}{|c|}{$<$ Status $>$ Completed $</$ Status $>$} \\
\hline \multicolumn{4}{|c|}{$<\mathrm{ICD}>\mathrm{F} 03</ \mathrm{ICD}>$} \\
\hline \multicolumn{4}{|c|}{$<$ Text $>$ Ausschluss von Demenz $</$ Text $>$} \\
\hline \multicolumn{4}{|c|}{$<$ Diagnosesicherheit $>8</$ Diagnosesicherheit $>$} \\
\hline \multicolumn{4}{|c|}{$<$ DiagnosesicherheitCode $>$ A $</$ DiagnosesicherheitCode $>$} \\
\hline \multicolumn{4}{|c|}{$<$ Diagnuse $>-<$ Diagnuse $>$} \\
\hline \multicolumn{4}{|c|}{$<$ Datum $>20101005</$ Datum $>$} \\
\hline \multicolumn{4}{|c|}{$<$ Status $>$ Completed $</$ Status $>$} \\
\hline \multicolumn{4}{|c|}{$<\mathrm{ICD}>\mathrm{E} 14.74</ \mathrm{ICD}>$} \\
\hline \multicolumn{4}{|c|}{$<$ Text $>$ Diabetisches Fußsyndrom $</$ Text $>$} \\
\hline \multicolumn{4}{|c|}{$<$ Diagnosesicherheit $>8<$ Diagnosesicherheit $>$} \\
\hline \multicolumn{4}{|c|}{$<$ DiagnosesicherheitCode $>\mathrm{G}</$ DiagnosesicherheitCode $>$} \\
\hline \multicolumn{4}{|l|}{$</$ Diagnose $>$} \\
\hline$</$ Diagnosen $>$ & & & \\
\hline
\end{tabular}

Abb. 7 Datenstruktur des AIS und Rückgabe des AIS-Adapters

möglich. Abbildung 8 illustriert die Benutzeroberfläche der eÜberweisung mit Überweisungsdaten eines Patienten mit Diabetes Mellitus Typ 2.

\subsection{HL7-CDA-Wrapper}

Die Interoperabilitätskomponente greift auf die Schnittstellen der Anwendungskomponente zu, um einen Datensatz, der nach fallbezogenen Kriterien aufbereitet wurde, in ein interoperabel nutzbares Format zu überführen. Im Falle der
eÜberweisung muss das AIS des Empfängers die Daten fehlerfrei verarbeiten können und der Nutzer des IS die Inhalte im Sinne des Absenders interpretieren. Als interoperables Austauschformat wurde zu diesem Zweck ein einheitlicher Überweisungsträger auf Basis HL7 CDA konzipiert, das auf dem elektronischen Arztbrief des Verbands der Hersteller von IT-Lösungen für das Gesundheitswesen (VHitG) basiert (VHitG 2006). Die Stammdaten von Arzt und Patient werden in den HL7-Header des Über- 


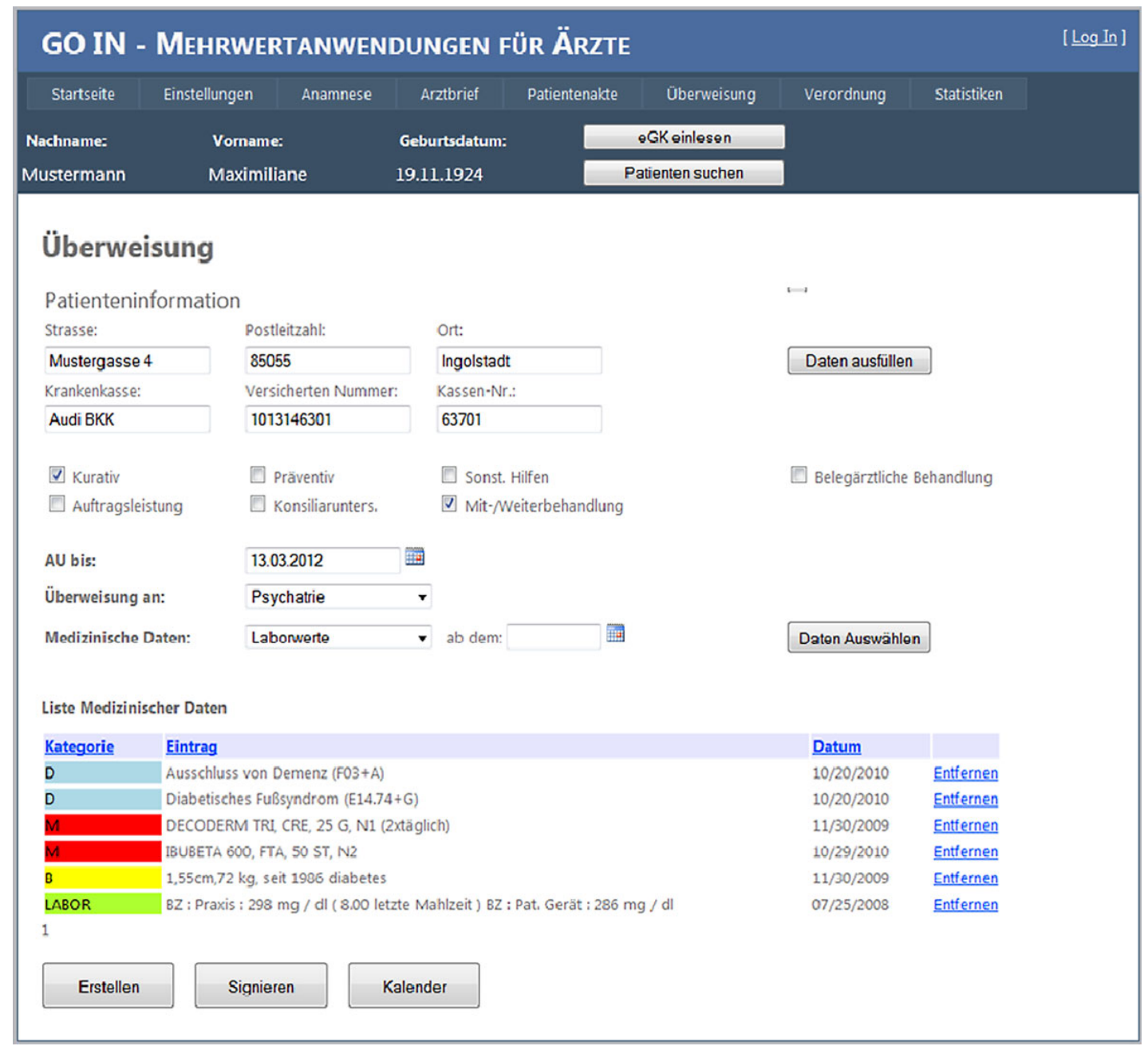

Abb. 8 Die eÜberweisung

weisungsträgers überführt, der für weitere digitale Formulare wiederverwendbar ist. Die nach ICD und PZN aufbereiteten medizinischen Daten werden mit einem LOINC-Header versehen und als CDA-Level-3-Komponenten in den CDA-Body des Dokuments überführt (VHitG 2006). Die übrigen Datenelemente wurden in Level-2-Komponenten aufbereitet; eine übergreifende Maschinenlesbarkeit ist für diese Daten auf Grund des Adapters nicht möglich. Die Schnittstellen in der Komponente wurden so benannt, dass der Aufruf auch für CCR-Records oder Verordnungen der gematik angepasst werden kann. Mit Hilfe eines übergreifenden Stylesheets wird sichergestellt, dass alle Nutzer des Dokuments denselben visuellen Eindruck haben. Es können weitere Elemente hinzugefügt werden, die auf den Papierformularen zurzeit nicht vorgesehen oder verwendbar sind, z.B. Anamnese- oder Bilddokumente (Abb. 9).

\subsection{Signaturkomponente}

Um sicherzustellen, dass der Nutzer der MWA zur Erstellung einer Überweisung befähigt ist, muss eine elektronische Signatur mit einem Heilberufsausweis (HBA) erstellt werden, bevor die Transaktion abgeschlossen werden kann. Die Signaturkomponente greift auf die Interoperabilitätsschicht zu, um das HL7CDA-Dokument zu erhalten. Dann wird der private Schlüssel des Hausarztes aus dem HBA ausgelesen, wobei die ausreichende Autorität festgestellt wird, indem der Arzt seine PIN eingibt (gematik 2009c). Die qualifizierte elektronische Signatur wird über die Konnektorschnittstelle signDocument erstellt. Die Signaturkomponente erzeugt nach der Signatur eine TAN, die Patienten und Arzt- helfer zur Reservierung eines Termins bei der angegebenen Facharztgruppe berechtigt. Nach diesem Schritt werden das Dokument und die TAN in einem Austauschverzeichnis abgelegt, das von der lokalen Komponente des Terminmanagers ausgelesen werden kann. Auf Seite des Empfängers kann die Signatur gegen das Zertifikat des Hausarztes verifiziert werden, um die Echtheit zu garantieren (gematik 2009b). Die Komponente nutzt vorwiegend Webservices der gematik und kann so auch für weitere digitale Formulare in anderen MWA verwendet werden.

\subsection{Steuerungskomponente}

Das Praxisnetz strebt eine Verkürzung der Wartezeiten für Facharzttermine an. Die freien Termine für eine Facharztbehandlung werden daher vom Netz zentral bereitgestellt, wenn sie vorher von den 


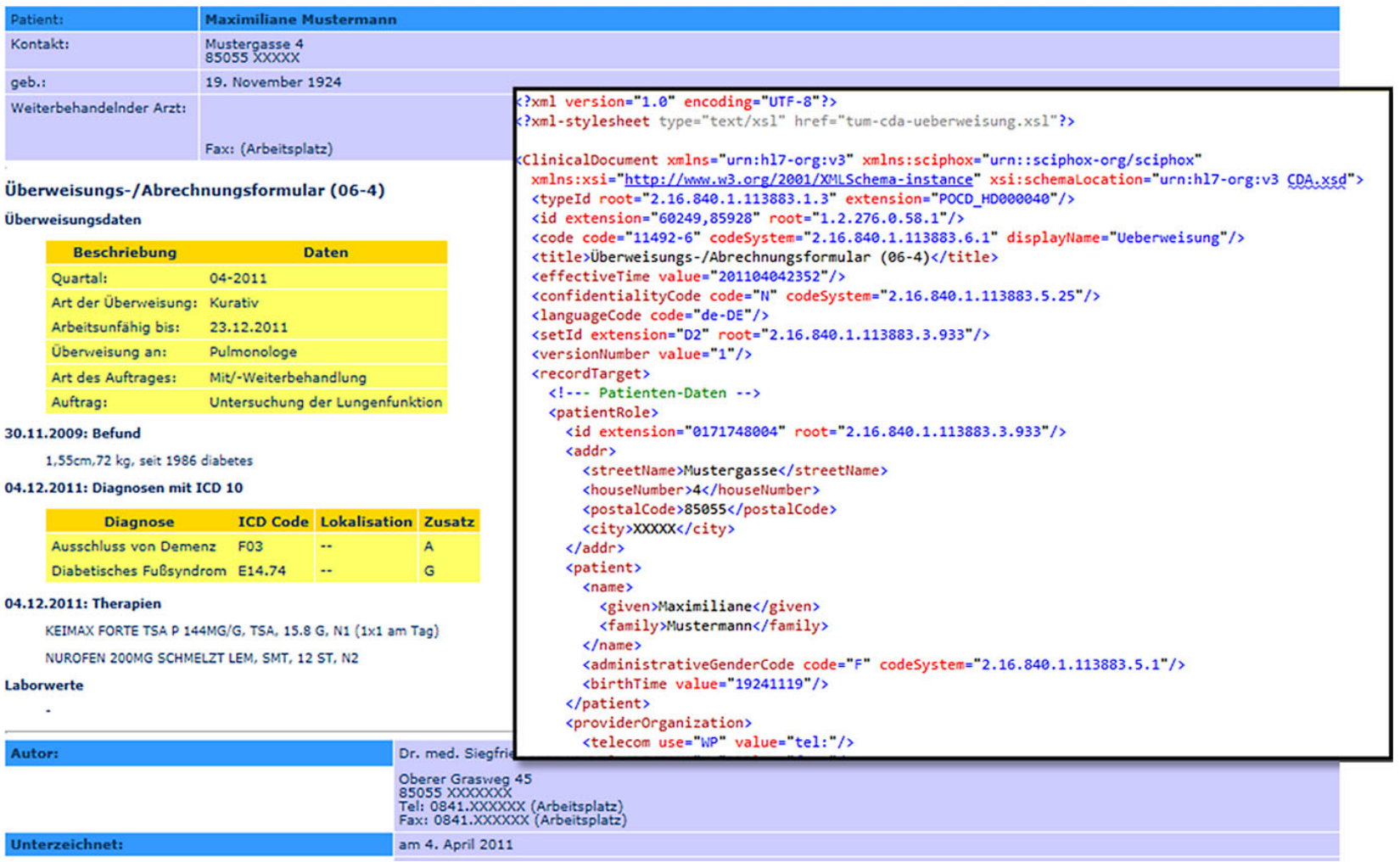

Abb. 9 HL7-CDA-Ansicht der eÜberweisung
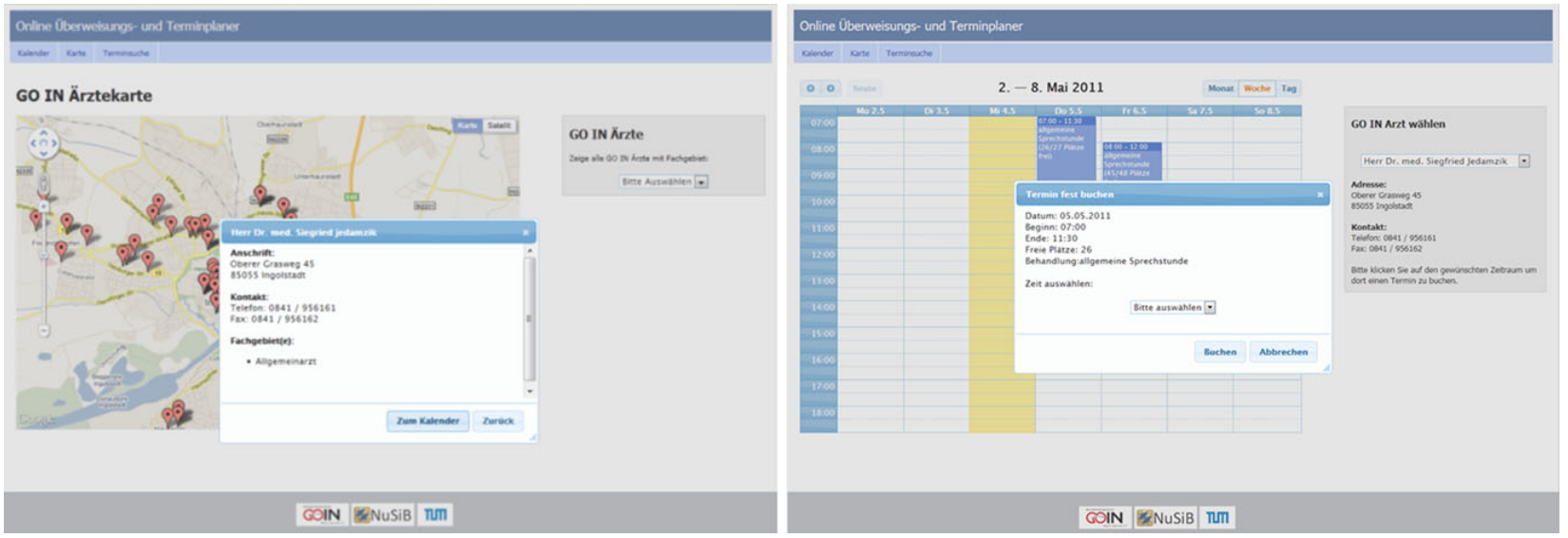

\section{Abb. 10 Netzdatenauswahl und Terminreservierung}

Ärzten freigegeben wurden. So können Patienten und Praxishelfer die verfügbaren Termine nach Kriterien wie Zeitpunkt, Entfernung oder Behandlungsart darstellen. Dies soll eine optimale Verteilung der freien Termine auf die nachfragenden Patienten ermöglichen und die telefonische Koordination minimieren. $\mathrm{Zu}$ diesem Zweck wurde der Webservice createAppointment geschaffen, der mit den Parametern Zeitslot, Behandlungsart und Stammdaten eines Arztes neue Terminslots erzeugt. Über die Schnitt- stelle getAppointments können freie Termine abgerufen werden, die dann in der Kalenderkomponente oder in einer Übersicht angezeigt werden. Es können optionale Einschränkung gemacht werden. Ortsradius, Terminspanne, Behandlungsart und Stammdaten des Arztes sind mögliche Parameter. Die Visualisierung wird bei der Vermittlung von elektronischen Überweisungen verwendet, etwa indem ein freier Termin innerhalb der nächsten sieben Tage gesucht wird. Die Steuerungskomponente fungiert als
Nutzerschnittstelle, die Aufrufe automatisiert, indem die Parameter automatisch übergeben werden, wenn der Kalender eines Arztes auf der Karte gewählt wird oder eine Terminspanne und eine Behandlungsart von Nutzern angegeben wird (Abb. 10).

\subsection{Terminauswahl}

Die Terminkomponente ruft die Steuerungsdatenkomponente auf, um freie Terminslots zu erhalten. Die Komponen- 
te für Patienten läuft auf einem Server des Praxisnetzes und ist über das Internet erreichbar. In den lokalen Praxisanwendungen können Termine der eigenen Arztpraxis angelegt, freigegeben und reserviert werden. Eine Freigabe enthält die Facharztgruppe, die Behandlungsart, den verfügbaren Zeitrahmen und die Länge eines einzelnen Termins. Wird ein Termin freigegeben, erfolgt eine Synchronisation mit dem zentralen Termindienst. Durch Eingabe der TAN können alle verfügbaren Termine einer bestimmten Facharztgruppe im zentralen Dienst eingesehen und reserviert werden. Dieser Schritt kann sowohl durch das Praxispersonal an einer lokalen MWA durchgeführt werden als auch von Patienten selbst an der zentralen MWA. Durch die Reservierung eines Termins wird die TAN gesperrt. Bei Absage eines Termins durch den Patienten oder das Praxispersonal wird die TAN wieder aktiviert, wenn der Zeitpunkt der Absage innerhalb desselben Quartals liegt.

\subsection{Verschlüsselung}

Zur adäquaten Sicherung der Kommunikation wurde die hybride Verschlüsselung der Überweisungsträger durch den Konnektor gewählt (gematik 2009b). Nach der Terminreservierung wird das Zertifikat des weiter behandelnden Arztes in die MWA geladen und das Überweisungsformular mit diesem verschlüsselt und in einem Austauschdienst des Terminservers temporär abgelegt, bis sie von der lokalen MWA des Zielarztes abgerufen wird. Zusätzlich wird ein Termindatensatz auf dem zentralen Server abgelegt und vorgehalten. Dieser enthält nur den vereinbarten Termin, die Facharztzuordnung und ein Patientenpseudonym. Dieses Pseudonym wird erst beim Besuch der Praxis auf dem lokalen System des Zielarztes mit den Personendaten von der eGK versehen. Anschließend kann der Facharzt die eÜberweisung mit seinem HBA entschlüsseln und die Inhalte verwenden. So ist es bei einem Hackerangriff nicht möglich, die Termindaten realweltlichen Personen zuzuordnen (Dünnebeil et al. 2011).

\section{Implikationen}

Der Bedarf an integrierten und regional angepassten Behandlungsprozessen bei gleichzeitiger Ablehnung von zentralen
Patientenakten im deutschen Gesundheitswesen bedingt die Entwicklung von verteilten Softwareanwendungen. Institutionsübergreifende Prozesse auf Basis der bestehenden IS bereitzustellen ist aufgrund der großen Diversität proprietärer und geschlossener Primärsysteme jedoch oft nicht möglich. Angepasste Prozesse können durch verteilte MWA, wie der Anwendungsfall der eÜberweisung gezeigt hat, strukturiert konzipiert und implementiert werden. Die Basisdienste der TI können dabei für wichtige Aufgaben genutzt werden. Entwickler können zukünftig in strukturierter Art und Weise solche MWA implementieren, wenn sie den Vorgaben der vorgestellten Architektur folgen. Auf jeder Schicht können für wohldefinierte Funktionseinheiten Standards festgelegt und Anforderungen erhoben werden, um wichtige Kriterien für medizinische Anwendungen zu berücksichtigen. Etablierte Komponenten können in ähnlichen Anwendungsfällen wiederverwendet werden, um Entwicklungsaufwände zu reduzieren. Langfristig kann so ein umfangreiches Service-Ecosystem (Barros und Dumas 2006) um die eGK entstehen, das etablierte Konzepte aus der Informatik umsetzt. Die Entwicklung in der Mobilfunkbranche kann dabei als eine Referenz angesehen werden, wenngleich Sicherheits- und Datenschutzaspekte im Gesundheitswesen einen höheren Stellenwert einnehmen. Die Telematikinfrastruktur muss in MWA nicht in vollem Umfang genutzt werden; sie stellt in vielen Bereichen vielmehr eine nützliche Unterstützung bereit, um Anforderungen umzusetzen, die von vielen Leistungserbringern gefordert werden. Auf die dauerhafte zentrale Speicherung von Patientendaten kann bei Telematikanwendungen verzichtet werden, wenn ein Ansatz mit verteilten MWA gewählt wird. Der Nutzen kann so in den Vordergrund gestellt werden, da wichtige Sicherheitsaspekte bereits in die Entwicklung der Architektur eingeflossen sind.

Der vorliegende Beitrag liefert erste Eckpunkte einer Referenzarchitektur für MWA der TI, es bedarf jedoch der Analyse weiterer medizinischer Versorgungsprozesse, um eine größere Bandbreite von Anforderungen einfließen zu lassen. Eine Evaluierung und iterative Verfeinerung einer Referenzarchitektur kann über Anwendungen im Feld erfolgen, wenn der Ausbau der TI dies zulässt. Ferner ergeben sich viele technische und organisatorische Herausforderungen bei der
Umsetzung der einzelnen Architekturschichten, die detaillierter wissenschaftlicher Reflexion bedürfen. Der vorliegende Artikel konnte diese Aspekte nur in Teilen behandeln, da Wert auf eine ganzheitliche Darstellung von MWA der TI gelegt wurde. Der Fokus lag auf einer umfassenden Einführung und Motivation des Themas, der Erhebung und Strukturierung von grundlegenden Anforderungen und der Illustration einer Beispielanwendung. So konnte die Grundlage für eine differenziertere Diskussion der Gesundheitstelematik gelegt werden, indem Konzepte dargestellt wurden, die über die bekannten Fachanwendungen hinausgehen.

\subsection{Theoretischer Beitrag}

Der Beitrag erhebt und strukturiert wichtige Anforderungen des Gesundheitswesens in einer übergreifenden Architektur für MWA. Die Aspekte Einbindung von Altsystemen, Interoperabilität, $\mathrm{Au}-$ torisierung, Prozesssteuerung, Nachrichtenvermittlung und Sicherheit, die schon in vorherigen Architekturansätzen untersucht wurden, werden in einer abstrakten Architektur vereinigt und in einen strukturierten Zusammenhang gebracht. So entsteht eine abstrakte Designtheorie für verteilte MWA im Gesundheitswesen. Die Architektur reiht sich damit in die Beiträge zum Softwareengineering ein, die in diesem Zusammenhang Systementwicklungsansätze für verschiedene Domänen anbieten kann, wenn Einbindung von Altsystemen, rechtsverbindliche Signaturen, Interoperabilität, Trennung von Produktiv- und Steuerungsdaten und objektivierte und verschlüsselte Vermittlung von Bedeutung sind. Die Verifikation der Architektur, der MWA zur eÜberweisung, stellt ferner einen frühen Prototypen für Telematikanwendungen dar, der Anforderungen auf Basis der technischen Infrastruktur der deutschen Gesundheitstelematik umsetzt, wie es in Hevner u. a. (2004) für neue Technologien gefordert wird.

\subsection{Praktische Implikationen}

Softwareentwickler erhalten mit diesem Beitrag eine Diskussionsgrundlage für wichtige Architekturentscheidungen, auf deren Grundlage sie konkrete Designentscheidungen treffen können. Nutzenpotenziale können auf Basis einer theoretisch fundierten Architektur umgesetzt 


\section{Zusammenfassung / Abstract}

Sebastian Dünnebeil, Ali Sunyaev, Jan Marco Leimeister, Helmut Krcmar

\section{Modulare Softwarearchitektur für Mehrwertanwendungen der deutschen Gesundheitstelematik}

Mehrwertanwendungen der elektronischen Gesundheitskarte sollen Patientenorientierung sowie Qualitäts- und Effizienzsteigerungen im Gesundheitswesen ermöglichen. Die Dienste der Gesundheitstelematik, bei der die elektronische Gesundheitskarte nur eine Teilkomponente ist, können dabei beliebig eingebunden werden, etwa um Sicherheitsstandards und Interoperabilität zu gewährleisten. Der gestaltungsorientierte Beitrag präsentiert die Entwicklung und Evaluierung einer Softwarearchitektur für Mehrwertanwendungen. Um die Akzeptanz der Nutzer zu steigern, wurden die Architekturanforderungen direkt bei den Leistungserbringern erhoben. Die Architektur wird anhand des elektronischen Überweisungsmanagements illustriert und evaluiert. Es werden typische Funktionseinheiten von medizinischen Abläufen in sieben Schichten abstrahiert. Die Instanziierung zeigt eine strukturierte Anwendung mit übergreifenden Sicherheitsstandards, die Interoperabilität zwischen heterogenen Bestandssystemen und eine übergreifende Steuerung durch Ärzteverbünde ermöglicht. Die Architektur bietet erstmalig im deutschen Gesundheitswesen die Möglichkeit, eine Vielzahl von Mehrwertanwendungen systematisch, einfach, sicher und zuverlässig zu spezifizieren und zu entwickeln, um sie über die Telematikinfrastruktur zur Verfügung zu stellen.

Schlüsselwörter: Gesundheitskarte, Telematikinfrastruktur, Mehrwertanwendungen, Mehrwertdienste, Referenzarchitektur, Gesundheitswesen, Design research

\section{Modular Architecture of Value-Added Applications for German Healthcare Telematics}

Value-added applications of the German healthcare telematics aim at patient orientation as well as quality and efficiency improvements in the healthcare sector. Telematics services can be utilized in many ways, e.g., to guarantee security standards and interoperability. The electronic health card is merely one example. The design science oriented article presents the development and evaluation of a software architecture for value-added applications. In order to achieve better user acceptance, the architectural requirements were derived directly from the caregivers. The architecture is illustrated and evaluated by the example of electronic referral management. Typical functional units of medical processes are modeled in seven layers. The instantiation shows a structured application with high security standards which enables interoperability between heterogeneous existing systems and extensive control by physicians' cooperatives. The architecture offers, for the first time in German healthcare, the opportunity to safely and reliably specify and develop a variety of value-added applications which can be provided via the telematics infrastructure.

Keywords: Electronic health card, Healthcare telematics, Value-added applications, Value-added services, Software architecture, Design research werden, deren Eckpunkte direkt aus Anforderungen von Leistungserbringer abgeleitet wurden. So wird der Forderung nach stärkerer Integration der Nutzer in die Telematik Rechnung getragen. Ferner sollte die Entwicklung von MWA stärker in den Fokus der Aktivitäten von Behörden und gematik gerückt werden, um die Potenziale der eGK aufzuzeigen, die sich jenseits der vieldiskutierten Fachanwendungen ergeben. Die Entwicklung der eÜberweisung stieß bei den beteiligten Ärzten auf großes Interesse, da sie gegenwärtig viele Ressourcen für die Koordination von Terminen aufwenden müssen. Die Darstellung der Einführung der eGK als eine notwendige Voraussetzung für solche Prozesse, die ohne elektronische Signatur und einheitliche Verschlüsselung nicht umzusetzen sind, kann die Wahrnehmung der eGK erheblich verbessern.

Weitere Anwendungen wurden auf Basis des Architekturmusters diskutiert und umgesetzt, aber in diesem Artikel nicht in dargestellt (vgl. Dünnebeil et al. 2011). Die Erfassung von Vitalparametern durch Patienten und deren Kommunikation zu niedergelassenen Ärzten im Rahmen von TelemonitoringProgrammen wurde untersucht, auch die strukturierte digitale Kommunikation zwischen Ärzten und Apothekern konnte in einer MWA zum Verordnungsmanagement implementiert werden, die der vorgestellten Referenzarchitektur folgt. Es zeigten sich erheblich Potenziale zur Wiederverwendung von bestehenden Modulen und eine wohldefinierte Struktur der Programme.

\section{Literatur}

Allensbach (2010) ifD-Umfrage 5283. Der Einsatz von Telematik und Telemedizin im Gesundheitswesen. Allensbach 2010

Arsanjani A, Allam A (2006) Service-oriented modeling and architecture for realization of an SOA. In: IEEE International conference on services computing, Chicago, S 521

Audi BKK (2011) Audi BKK und das Praxisnetz GO IN unterzeichnen neuen Versorgungsvertrag - Audi BKK. http://www.audibkk. de/Presse-Archiv.38+M5022316c79a.0. html. Abruf am 2011-12-13

Barros AP, Dumas M (2006) The rise of web service ecosystems. IT Professional 8(5):3137

Bernnat R (2006) Kosten-Nutzen-Analyse der Einrichtung einer Telematik-Infrastruktur im deutschen Gesundheitswesen. Booz Allen Hamilton $\mathrm{GmbH}$, Düsseldorf

BITKOM (2009) Position der Leistungserbringer zur Neuausrichtung der Einführung eGK/Telematikinfrastruktur (Gesundheitskarte) - BITKOM. http://www.bitkom.org/ de/themen/37207_62030.aspx. Abruf am 2011-12-13 
Blobel B (2004) Authorisation and access control for electronic health record systems. International Journal of Medical Informatics 73(3):251-257

BMG (2005) The German eHealth strategy (target and strategy, concept, legal framework, activities/roll-out plan, costs and return of investment. European perspective). Berlin

BRD (2004a) Sozialgesetzbuch (SGB) Fünftes Buch, Gesetzliche Krankenversicherung, $\S 291 \mathrm{a}$

BRD (2004b) Sozialgesetzbuch (SGB) Fünftes Buch, Gesetzliche Krankenversicherung, $\S 140 a$

Buschmann F, Meunier R, Rohnert H, Sommerlad P, Stal M (1996) Pattern-oriented software architecture. Wiley, New York

Chandramouli R (2001) A framework for multiple authorization types in a healthcare ap plication system. In: Proc 17th annual computer sec appl conf, Los Alamitos, p 137

Dehling T, Sunyaev A (2012) Information security of patient-centered services utilising the German nationwide health information technology infrastructure. In: 3rd USENIX workshop on health security and privacy (HealthSec 2012), co-located with the 21st USENIX security symposium (USENIX Security '12), Bellevue. https://www.usenix.org/conference/ healthsec12/information-security-patientcentered-services-utilising-germannationwide. Abruf am 2012-10-16

Dern G (2009) Management von ITArchitekturen: Leitlinien für die Aus richtung, Planung und Gestaltung von Informationssystemen. Vieweg + Teubner, Wiesbaden

Dijkstra EW (1968) The structure of the THE multiprogramming system. Communications of the ACM 11(5):341-346

Dinh M, Chu M (2006) Evolution of health information management and information technology in emergency medicine. Emergency Medicine Australasia 18(3):289-294

Dünnebeil S, Sunyaev A, Blohm I, Leimeister JM, Krcmar H (2010a) Do German physicians want electronic health services? A characterization of potential adopters and rejecters in German ambulatory care. In: 3rd international conf on health informatics 2010, Valencia, S 202-209

Dünnebeil $S$, Sunyaev A, Leimeister JM Krcmar H (2010b) Strategies for development and adoption of EHR in German ambulatory care. In: 4th IEEE int conference on pervasive computing technologies for healthcare, Munich. doi:10.4108/ ICST.PERVASIVEHEALTH2010.8887

Dünnebeil S, Kaletsch A, Jedamzik S, Sunyaev A, Leimeister JM, Krcmar H (2011) Prozessdigitalisierung durch Mehrwertanwendungen der eGK am Beispiel der elektronischen Überweisung. In: perspeGKtive 2011 - In novative und sichere Informationstechnologie für das Gesundheitswesen von morgen, Darmstadt

Dünnebeil S, Sunyaev A, Blohm I, Leimeister JM, Krcmar H (2012) Determinants of physicians' technology acceptance for ehealth in ambulatory care. International Journal of Medical Informatics 81(11):746760. doi:10.1016/j.ijmedinf.2012.02.002

Eichelberg M, Aden T, Riesmeier J Jr, Dogac A, Laleci GB (2005) A survey and analysis of electronic healthcare record standards. ACM Computing Surveys 37(4):277-315

Fayn J, Ghedira C, Telisson D, Atoui H, Placide J, Simon-Chautemps L, Chevalier P, Rubel $P$ (2003) Towards new integrated information and communication infrastructures in e-health. Examples from cardiology. In: Computers in cardiology, Lyon, S 113-116

Ferranti JM Musser RC Kawamoto $\mathrm{K}$, Ham mond WE (2006) The clinical document architecture and the continuity of care record: a critical analysis. Journal of the American Medical Informatics Association 13(3):245-252

Fettke P, Loos P (2002) Methoden zur Wiederverwendung von Referenzmodellen Übersicht und Taxonomie. In: Multikonferenz Wirtschaftsinformatik, Nürnberg, S 933

Fraunhofer (2005) Spezifikation der Lösungsarchitektur zur Umsetzung der Anwendungen der elektronischen Gesundheitskarte. Fraunhofer ISST, IAO, SIT, FuE-Projekt "Lösungsarchitektur", Berlin

Frießem P, Kalmring D, Reichelt P (2005) Lösungsarchitektur für die Einführung der elektronischen Gesundheitskarte und der auf ihr basierenden Anwendungen. WIRTSCHAFTSINFORMATIK 47(3):180-186

gematik (2009a) Einführung der Gesundheitskarte - Gesamtarchitektur. Berlin

gematik (2009b) Einführung der Gesundheitskarte - Konnektorspezifikation. Berlin gematik (2009c) Einführung der Gesundheitskarte - Übergreifendes Sicherheitskonzept. Berlin

gematik (2012) Anwendungen der eGK http://www.gematik.de/cms/de/egk_2/ anwendungen/anwendungen_1.jsp. Abruf am 2012-05-15

Gregor S (2006) The nature of theory in information systems. MIS Quarterly 30(3):611642

Gritzalis D, Lambrinoudakis C (2004) A security architecture for interconnecting health information systems. International Journal of Medical Informatics 73(3):305-309

Hasselbring W (2000) Information system integration. Communications of the ACM 43(6):32-38

Hevner A, March S, Park J (2004) Design science in information systems research. Management Information Systems Quarterly 28(1):75-105

Holzer A, Ondrus J (2011) Mobile application market: a developer's perspective. Telematics and Informatics 28(1):22-31

Hornung G, Goetz CFJ, Goldschmidt AJW (2005) Die künftige TelematikRahmenarchitektur im Gesundheitswesen. Recht, Technologie, Infrastruktur und Ökonomie. WIRTSCHAFTSINFORMATIK 47(3):171-179

Jacobs K, Schulze S (2004) Wettbewerbsperspektiven integrierter Versorgung in der gesetzlichen Krankenversicherung. Gesundheitsökonomische Beiträge 44:89-110

Kaletsch A, Sunyaev A (2011) Privacy engineering: personal health records in cloud computing environments. In: Proc 32th international conference on information systems, Shanghai, Paper 2

KBV (2010) Online-Anbindung. http://www. kbv.de/12629.html. Abruf am 2012-01-12

KBV (2011a) Installationsstatistik - Systeme. In: http://www.kbv.de/ita/4299.html. Abruf am 2012-01-12

KBV (2011b) Anderung der Vereinbarung über Vordrucke für die vertragsärztliche Versorgung. Deutsches Ärzteblatt 108(50):2739

Kirn S (2005) Gesundheitsinformatik - Informationssysteme für das Gesundheitswesen von morgen. WIRTSCHAFTSINFORMATIK 47(3):165-166

Köbler F, Fähling J, Krcmar H (2010) ITGovernance und IT-Entscheidertypen in deutschen Krankenhäusern: Eine empirische Untersuchung unter KrankenhausIT-Leitern. WIRTSCHAFTSINFORMATIK 52(6):353-365

Kumbruck C (oJ) Digitale Signaturen und Vertrauen. In: https://eldorado.tudortmund.de/handle/2003/28314. Abruf am 2012-03-12

Lappé JM, Muhlestein JB, Lappé DL, Badger RS (2004) Improvements in 1-year cardiovascular clinical outcomes associated with a hospital-based discharge medication program. Annals of Internal Medicine 141(6):446-453

Marsic I (2001) An architecture for heterogeneous groupware applications. In: Int conf software engineering 2001, Washington, S 475-484

Mauro C, Sunyaev A, Leimeister J, Krcmar H (2009) Serviceorientierte Integration Medizinischer Geräte - Eine State of the Art Analyse. In: Proc Wirtschaftsinformatik 2009. Wien, S 119-128

Mercuri RT (2004) The HIPAA-potamus in healthcare data security. Communications of the ACM 47(7):25-28

Mertens P (2012) Schwierigkeiten mit ITProjekten der Öffentlichen Verwaltung - Neuere Entwicklungen. InformatikSpektrum 32(1):42-49. doi:10.1007/ s00287-008-0310-9

Neuhaus J, Deiters W, Wiedeler M (2006) Mehrwertdienste im Umfeld der elektronischen Gesundheitskarte. Informatik-Spektrum 29(5):332-340

O'Brien KJ (2010) Google data admission angers European officials. The New York Times

Ouksel AM, Sheth A (1999) Semantic interoperability in global information systems. ACM SIGMOD Record 28(1):5-15. doi:10.1145/309844.309849

Papazoglou MP (2003) Service-oriented computing: concepts, characteristics and directions. In: Fourth int conf on web information systems engineering, Rome, S 3-12

Pedersen S, Hasselbring W (2004) Interoperabilitat für Informationssysteme im Gesundheitswesen auf Basis medizinischer Standards. Informatik - Forschung und Entwicklung 18(3-4):174-188

Riedl B, Grascher V, Neubauer T (2008) A secure e-health architecture based on the appliance of pseudonymization. Journal of Software 3(2):23-32

Rindfleisch TC (1997) Privacy, information technology, and healthcare. Communications of the ACM 40(8):92-100

Rivest RL, Shamir A, Adleman L (1978) A method for obtaining digital signatures and public-key cryptosystems. Communications of the ACM 21(2):120-126

Rohner P, Winter R (2008) Was kann die Wirtschaftsinformatik dazu beitragen, E-Health voran zu bringen? WIRTSCHAFTSINFORMATIK 50(4):330-334

Sackett DL, Rosenberg WMC, Gray JAM, Haynes RB, Richardson WS (1996) Evidence based medicine: what it is and what it isn't. British Medical Journal 312(7023):71-72

Sarv D, Rajiv K (2000) Information technology payoff in the health-care industry: a longitudinal study. Journal of Management Information Systems 16(4):41

Sawicki P (2005) Qualität der Gesundheitsversorgung in Deutschland. Medizinische Klinik 100(11):755-768. doi:10.1007/s00063005-1105-2

Schweiger A, Sunyaev A, Leimeister JM, Krcmar H (2007) Information systems and healthcare XX: toward seamless healthcare 
with software agents. Communications of AIS 19(1):692-710

Shaw M, Garlan D (1996) Software architec ture: perspectives on an emerging discipline. Prentice Hall, New York

Shekelle P, Morton SC, Keeler EB (2006) Evidence report - technology assessment. In: Costs and benefits of health information technology

Silber S (2006) Argumente für die Integrierte Versorgung als Regelversorgung in de Kardiologie. Clinical Research in Cardiology 95(80):ii37-ii40

Stanford V (2002) Pervasive healthcare applications face tough security challenges. IEEE Pervasive Computing 1(2):8-12

Stewart M, Brown JB, Donner A, McWhinney IR Oates J Weston WW Jordan (2000) The impact of patient-centered care on outcomes. Journal of Family Practice 49(9):796-804

Stolba N, Tjoa A (2006) The Relevance of Data Warehousing and Data Mining in the Field of Evidence-based Medicine to Support Healthcare Decision Making. International Journal of Computer Systems Science and Engineering 3(3):143-148

Sunyaev A, Chornyi D (2012) Supporting chronic disease care quality: design and implementation of a health service and its integration with electronic health records. ACM Journal of Data and Information Quality 3(2):3:1-3:21. doi:10.1145/2184442.2184443
Sunyaev A, Pflug J (2012) Risk evaluation and security analysis of the clinical area within the German health information infrastruc ture. Health and Technology 2(2):123-135. doi:10.1007/s12553-012-0016-5

Sweeney L (1997) Guaranteeing anonymity when sharing medical data, the Datafly system. In: Proc AMIA annual fall symposium, Nashville, S 51-55

Szyperski C, Gruntz D, Murer S (2002) Component software: beyond object-oriented programming. Pearson Education, Upper Saddle River

TKK (2009) 1, Branchenbarometer E-Health F.A.Z.-Institut für Management-, Markt- und Medieninformation, Frankfurt

Tsai WT, Fan C, Chen Y, Paul R, Chung J-Y (2006) Architecture classification for SOA-based applications. In: Proc 9th IEEE international symposium on object and component-oriented real-time distributed computing, Gyeongju, S 295-302

Tsiknakis M, Chronaki CE, Kapidakis S, Nikolaou C, Orphanoudakis SC (1997) An integrated architecture for the provision of health telematic services based on digital library technologies. International Journal on Digital Libraries 1(3):257-277

Tsiknakis M, Katehakis DG, Orphanoudakis SC (2002) An open, component-based information infrastructure for integrated health information networks. International Journal of Medical Informatics 68(1-3):3-26
Tuffs A (2010) Germany puts universal health e-card on hold. British Medical Journal 340(1):c171

TURBOMED (2012) Preisliste \& HzV, Bestellfaxe. http://www.turbomed.de/turbomed/ preisliste. Abruf am 2012-05-14

Urgaonkar B, Pacifici G, Shenoy P, Spreitzer M, Tantawi A (2007) Analytic modeling of multitier internet applications. ACM Trans Web 1(1). doi:10.1145/1232722.1232724

VHitG (2006) Arztbrief auf Basis der HL7 Clinical Document Architecture Release 2 für das deutsche Gesundheitswesen - Implementierungsleitfaden. http://download.vhitg.de/Leitfaden-

VHitG-Arztbrief-v150.pdf. Abruf am 201205-14

Vogel O, Arnold I, Chughtai A, Ihler E, Kehrer T, Mehlig U, Zdun U (2009) SoftwareArchitektur: Grundlagen - Konzepte. Spektrum, Heidelberg

Wegner P (1996) Interoperability. ACM Computing Surveys 28(1):285-287

Wu S, Chaudhry B, Wang J, Maglione M, Mojica W, Roth E, Morton SC, Shekelle PG (2006) Systematic review: impact of health information technology on quality, efficiency, and costs of medical care. Annals of Internal Medicine 144(10):742-752

Zimmermann H (1980) OSI reference model the ISO model of architecture for open systems interconnection. IEEE Transactions on Communications 28(4):425-432 